\title{
FREE-STREAM PRESERVING FINITE DIFFERENCE SCHEMES ON CURVILINEAR MESHES*
}

\author{
YAN JIANG ${ }^{\dagger}$, CHI-WANG SHU ${ }^{\ddagger}$, AND MENGPING ZHANG $§$ \\ Dedicated to Professor Stanley Osher on his seventieth birthday
}

\begin{abstract}
An important property for finite difference schemes designed on curvilinear meshes is the exact preservation of free-stream solutions. This property is difficult to fulfill for high order conservative essentially non-oscillatory (WENO) finite difference schemes. In this paper we explore an alternative flux formulation for such finite difference schemes [5] which can preserve free-stream solutions, based on the numerical technique for the metric terms [13], which can be applied to this alternative flux formulation but is difficult to be applied to the standard finite difference formulation. Free-stream and vortex preservation properties are investigated, and comparison with standard finite difference WENO schemes is made. Theoretical derivation and numerical results show that the finite difference WENO schemes based on the alternative flux formulation can preserve free-stream and vortex solutions on both stationary and dynamically generalized coordinate systems, hence giving much better performance than the standard finite difference WENO schemes for such problems.
\end{abstract}

Key words. High order finite difference scheme, weighted essentially non-oscillatory scheme, curvilinear meshes.

AMS subject classifications. 65M06.

1. Introduction. In this paper, we are interested in high order accurate conservative finite difference weighted essentially non-oscillatory (WENO) schemes on curvilinear meshes. Conservative finite difference schemes share many advantages of finite volume schemes, such as conservation and high order accuracy, yet they are much less expensive than finite volume schemes in multi-dimensions. The first WENO scheme was a third order finite volume scheme designed in [6]. Standard finite difference WENO schemes $[4,1,9]$ use the idea of reconstruction, which is the main relevant WENO procedure for designing both conservative finite volume and conservative finite difference schemes to solve hyperbolic conservation laws. In these standard finite difference WENO schemes, the derivative $f(u)_{x}$ is approximated by a numerical flux difference, and the numerical flux is based on the reconstruction of the physical flux $f(u)$ rather than on the solution $u$ as in finite volume methods.

When finite difference schemes are applied to curvilinear meshes (also referred to as generalized coordinate systems), free-stream preservation is an important property because errors from nonpreserved free-stream hide small physical oscillations, such as turbulent flow structures or aero-acoustic waves. Research $[13,7]$ shows that if the free-stream preservation condition is not satisfied, it will cause large errors and even lead to numerical instabilities for high-order schemes.

For the numerical fluxes in standard finite difference WENO schemes, since the reconstruction is performed directly on the flux values $\left\{f\left(u_{i}\right)\right\}$ (or $\left\{f^{+}\left(u_{i}\right)\right\}$ and

\footnotetext{
*Received April 16, 2013; accepted for publication August 1, 2013.

†School of Mathematical Sciences, University of Science and Technology of China, Hefei, Anhui 230026, P. R. China (jiangy@mail.ustc.edu.cn).

${ }_{\ddagger}$ Division of Applied Mathematics, Brown University, Providence, RI 02912, USA (shu@dam. brown.edu). Research supported by AFOSR grant F49550-12-1-0399 and NSF grant DMS-1112700.

$\S$ School of Mathematical Sciences, University of Science and Technology of China, Hefei, Anhui 230026, P. R. China (mpzhang@ustc.edu.cn). Research supported by NSFC grants 11071234, 91130016 and 91024025.
} 
$\left\{f^{-}\left(u_{i}\right)\right\}$ with a flux splitting $\left.f(u)=f^{+}(u)+f^{-}(u)\right)$, not on the point values of the solution $\left\{u_{i}\right\}$, it is difficult to maintain free-stream solutions exactly in curvilinear meshes for multi-dimensional flow computation. This is because the fluxes in curvilinear coordinates involve metric derivatives, resulting in non-exact cancellations when nonlinear reconstructions are performed for different fluxes.

Visbal and Gaitonde [13] carefully studied the metric evaluation errors for highorder central type compact schemes and found that for the three-dimensional generalized coordinate systems, the schemes can preserve the free-stream condition if they use the same formulas for the evaluation of both the metric and convection terms by adopting a conservative form of those metric terms, originally proposed by Thomas and Lombard [11]. However, it is difficult to apply the technique in $[11,13]$ to the standard conservative finite difference WENO schemes which are highly nonlinear. In [7], the free-stream and vortex preservation properties of the standard finite difference WENO schemes on stationary curvilinear grids have been investigated. It was found that standard finite difference WENO schemes could preserve the free-stream condition in the Cartesian coordinate system, but not in the generalized coordinate system. In addition, the schemes in generalized grids have a rather large error arising from the metric terms on randomized grids and three-dimensional wavy grids. In [2], Cai and Ladeinder also mentioned the difficulty of maintaining free-stream conditions in generalized curvilinear coordinate systems for high order finite difference WENO schemes.

Recently, an alternative flux formulation for the conservative finite difference WENO scheme, originally proposed in [10], was systematically studied in [5]. In this method, the high order WENO interpolation procedure is applied to the solution $\left\{u_{i}\right\}$ itself rather than to the flux functions $\left\{f\left(u_{i}\right)\right\}$. In [5], several advantages of this alternative flux formulation, including the increased flexibility to use arbitrary monotone fluxes in the scalar case and arbitrary approximate Riemann solvers in the system case, as well as a narrower effective stencil when applying the Lax-Wendroff time discretization, were found. Another major advantage of this alternative flux formulation is that it allows us to apply the technique in $[11,13]$ to obtain the freestream preserving property for high order conservative finite difference schemes. This advantage will be fully investigated in this paper.

The organization of the remaining sections is as follows. In Section 2, we review the alternative flux formulation for finite difference WENO schemes introduced in [5], using the one-dimensional Euler system as an example. The numerical methods for the three-dimensional Euler equations on generalized meshes and the analysis of the free-stream preserving condition will be discussed in Section 3. In Section 4, extensive numerical examples are provided to demonstrate the free-stream and vortex preservation performance of the new method in comparison with the standard finite difference WENO schemes. Concluding remarks are given in Section 5 .

2. Alternative flux formulation in one-dimension. The following onedimensional Euler equations with uniform grids are used for the discussion of the scheme.

$$
\frac{\partial Q}{\partial t}+\frac{\partial F}{\partial x}=0,
$$

where

$$
\begin{aligned}
& Q=(\rho, \rho u, e)^{T} \\
& F=\left(\rho u, \rho u^{2}+p, u(e+p)\right)^{T} .
\end{aligned}
$$


On a uniform mesh $x_{i}=i \Delta x$, we would like to find a consistent numerical flux function

$$
\hat{F}_{i+\frac{1}{2}}=\hat{F}\left(Q_{i-r}, \ldots, Q_{i+s}\right),
$$

such that the flux difference approximates the derivative $F(Q(x))_{x}$ to $k$-th order accuracy

$$
\frac{1}{\Delta x}\left(\hat{F}_{i+\frac{1}{2}}-\hat{F}_{i-\frac{1}{2}}\right)=\left.F(Q(x))_{x}\right|_{x_{i}}+O\left(\Delta x^{k}\right) .
$$

The alternative flux formulation, first developed in [10] and extensively explored in [5] in the context of WENO interpolation, is given as follows

$$
\hat{F}_{i+\frac{1}{2}}=F_{i+\frac{1}{2}}+\sum_{\ell=1}^{[(r-1) / 2]} a_{2 \ell} \Delta x^{2 \ell}\left(\frac{\partial^{2 \ell}}{\partial x^{2 \ell}} F\right)_{i+\frac{1}{2}}+O\left(\Delta x^{r+1}\right)
$$

which guarantees $k=r$-th order accuracy in (4). The coefficients $a_{2 \ell}$ in (5) can be obtained through Taylor expansion and the accuracy constraint (4). To get an approximation with fifth order accuracy $(k=5$ in (4), thus $r=5$ in (5)), we have

$$
\hat{F}_{i+\frac{1}{2}}=F_{i+\frac{1}{2}}-\left.\frac{1}{24} \Delta x^{2} \frac{\partial^{2} F}{\partial x^{2}}\right|_{i+\frac{1}{2}}+\left.\frac{7}{5760} \Delta x^{4} \frac{\partial^{4} F}{\partial x^{4}}\right|_{i+\frac{1}{2}} .
$$

The first term of the numerical flux in (6) is approximated by

$$
F_{i+\frac{1}{2}}=h\left(Q_{i+\frac{1}{2}}^{-}, Q_{i+\frac{1}{2}}^{+}\right)
$$

with the values $Q_{i+\frac{1}{2}}^{ \pm}$obtained by a WENO interpolation based on neighboring point values $Q_{j}$ using the local characteristic variables $[8,3,9]$. The two-argument numerical function $h$ is based on an exact or approximate Riemann solver. For example, we can use the Godunov flux, the Lax-Friedrichs flux, the HLLC flux, etc. The exact Riemann solver is given by the exact solution of the conservation laws (1) with the following step function as the initial condition

$$
Q(x, 0)= \begin{cases}Q_{i+1 / 2}^{-} & x \leq 0 \\ Q_{i+1 / 2}^{+} & x \geq 0\end{cases}
$$

evaluated at the center $x=0$ for $t>0$ (this value is independent of $t$ ). The Godunov flux is then given as $h\left(Q_{i+\frac{1}{2}}^{-}, Q_{i+\frac{1}{2}}^{+}\right)=F(Q(0, t))$. The detailed formulation of the other numerical fluxes can be found in, e.g. [12].

The remaining terms of the numerical flux in (6) or (5) have at least $\Delta x^{2}$ in their coefficients, hence they only need lower order approximations and they are expected to contribute much less to spurious oscillations. It is the conclusion of [5] that these remaining terms can be approximated by simple central approximation or one-point upwind-biased approximation with suitable orders of accuracy, without using the more expensive WENO procedure. Also, since we use Runge-Kutta time stepping in this paper, rather than the Lax-Wendroff time discretization technique as in [5], we do not expand $\partial^{\ell} F / \partial x^{\ell}$ as functions of $Q$ and its spatial derivatives. We simply use fixedstencil interpolation on $F$ directly. The details will be shown in the next section. 


\section{Finite difference WENO scheme on curvilinear meshes.}

3.1. Three-dimensional Euler equations. In Cartesian coordinates $(x, y, z)$, the three-dimensional Euler equations are

$$
\frac{\partial Q}{\partial t}+\frac{\partial E}{\partial x}+\frac{\partial F}{\partial y}+\frac{\partial G}{\partial z}=0
$$

where

$$
\begin{aligned}
Q & =(\rho, \rho u, \rho v, \rho w, e)^{T}, \\
E & =\left(\rho u, \rho u^{2}+p, \rho u v, \rho u w, u(e+p)\right)^{T}, \\
F & =\left(\rho v, \rho u v, \rho v^{2}+p, \rho v w, v(e+p)\right)^{T}, \\
G & =\left(\rho w, \rho u w, \rho v w, \rho w^{2}+p, w(e+p)\right)^{T} .
\end{aligned}
$$

The governing equation (8) can be transformed in curvilinear coordinates $(\xi, \eta, \zeta, \tau)$

$$
\frac{\partial \tilde{Q}}{\partial \tau}+\frac{\partial \tilde{E}}{\partial \xi}+\frac{\partial \tilde{F}}{\partial \eta}+\frac{\partial \tilde{G}}{\partial \zeta}=0
$$

where

$$
\begin{aligned}
\tilde{Q} & =Q / J, \\
\tilde{E} & =\tilde{\xi}_{t} Q+\tilde{\xi}_{x} E+\tilde{\xi}_{y} F+\tilde{\xi}_{z} G, \\
\tilde{F} & =\tilde{\eta}_{t} Q+\tilde{\eta}_{x} E+\tilde{\eta}_{y} F+\tilde{\eta}_{z} G, \\
\tilde{G} & =\tilde{\zeta}_{t} Q+\tilde{\zeta}_{x} E+\tilde{\zeta}_{y} F+\tilde{\zeta}_{z} G .
\end{aligned}
$$

Here, we choose $\tau=t$. The inverse Jacobian $J^{-1}$ and the standard metrics are

$$
J^{-1}=\left|\frac{\partial(x, y, z, t)}{\partial(\xi, \eta, \zeta, \tau)}\right|=\left|\begin{array}{cccc}
x_{\xi} & x_{\eta} & x_{\zeta} & x_{\tau} \\
y_{\xi} & y_{\eta} & y_{\zeta} & y_{\tau} \\
z_{\xi} & z_{\eta} & z_{\zeta} & z_{\tau} \\
0 & 0 & 0 & 1
\end{array}\right|,
$$

$(11)\left\{\begin{array}{lll}\tilde{\xi}_{x}=\xi_{x} / J=y_{\eta} z_{\zeta}-y_{\zeta} z_{\eta}, & \tilde{\xi}_{y}=\xi_{y} / J=z_{\eta} x_{\zeta}-z_{\zeta} x_{\eta}, & \tilde{\xi}_{z}=\xi_{z} / J=x_{\eta} y_{\zeta}-x_{\zeta} y_{\eta}, \\ \tilde{\eta}_{x}=\eta_{x} / J=y_{\zeta} z_{\xi}-y_{\xi} z_{\zeta}, & \tilde{\eta}_{y}=\eta_{y} / J=z_{\zeta} x_{\xi}-z_{\xi} x_{\zeta}, & \tilde{\eta}_{z}=\eta_{z} / J=x_{\zeta} y_{\xi}-x_{\xi} y_{\zeta}, \\ \tilde{\zeta}_{x}=\zeta_{x} / J=y_{\xi} z_{\eta}-y_{\eta} z_{\xi}, & \tilde{\zeta}_{y}=\zeta_{y} / J=z_{\xi} x_{\eta}-z_{\eta} x_{\xi}, & \tilde{\zeta}_{z}=\zeta_{z} / J=x_{\xi} y_{\eta}-x_{\eta} y_{\xi},\end{array}\right.$

$$
\left\{\begin{array}{l}
\tilde{\xi}_{t}=\xi_{t} / J=-\left[x_{\tau} \tilde{\xi}_{x}+y_{\tau} \tilde{\xi}_{y}+z_{\tau} \tilde{\xi}_{z}\right] \\
\tilde{\eta}_{t}=\eta_{t} / J=-\left[x_{\tau} \tilde{\eta}_{x}+y_{\tau} \tilde{\eta}_{y}+z_{\tau} \tilde{\eta}_{z}\right] \\
\tilde{\zeta}_{t}=\zeta_{t} / J=-\left[x_{\tau} \tilde{\zeta}_{x}+y_{\tau} \tilde{\zeta}_{y}+z_{\tau} \tilde{\zeta}_{z}\right]
\end{array}\right.
$$

The time-derivative term in equation (9) is split as follows:

$$
(Q / J)_{\tau}=(1 / J) Q_{\tau}+Q(1 / J)_{\tau}
$$

Thus, the governing equation (9) can be written as

$$
\frac{\partial Q}{\partial \tau}=-J\left[\frac{\partial \tilde{E}}{\partial \xi}+\frac{\partial \tilde{F}}{\partial \eta}+\frac{\partial \tilde{G}}{\partial \zeta}+Q(1 / J)_{\tau}\right]
$$


In the uniform flow (free-stream flow) regions, i.e. when $Q, E, F$ and $G$ are constants, equation (13) is simplified as

$$
\frac{\partial Q}{\partial \tau}=-J\left[I_{t} Q+I_{x} E+I_{y} F+I_{z} G\right]
$$

where

$$
\left\{\begin{array}{l}
I_{x}=\left(\tilde{\xi}_{x}\right)_{\xi}+\left(\tilde{\eta}_{x}\right)_{\eta}+\left(\tilde{\zeta}_{x}\right)_{\zeta}, \\
I_{y}=\left(\tilde{\xi}_{y}\right)_{\xi}+\left(\tilde{\eta}_{y}\right)_{\eta}+\left(\tilde{\zeta}_{y}\right)_{\zeta}, \\
I_{z}=\left(\tilde{\xi}_{z}\right)_{\xi}+\left(\tilde{\eta}_{z}\right)_{\eta}+\left(\tilde{\zeta}_{z}\right)_{\zeta}, \\
I_{t}=\left(\tilde{\xi}_{t}\right)_{\xi}+\left(\tilde{\eta}_{t}\right)_{\eta}+\left(\tilde{\zeta}_{t}\right)_{\zeta}+(1 / J)_{\tau} .
\end{array}\right.
$$

As discussed in [14], $I_{x}=I_{y}=I_{z}=0$ constitutes a differential statement of surface conservation, often termed the surface conservation law (SCL). $I_{t}=0$ is the volume conservation law (VCL). By substituting (10)-(12) into (15), we can see

$$
\left\{\begin{aligned}
I_{x}= & \left(y_{\eta} z_{\zeta}-y_{\zeta} z_{\eta}\right)_{\xi}+\left(y_{\zeta} z_{\xi}-y_{\xi} z_{\zeta}\right)_{\eta}+\left(y_{\xi} z_{\eta}-y_{\eta} z_{\xi}\right)_{\zeta}=0 \\
I_{y}= & \left(z_{\eta} x_{\zeta}-z_{\zeta} x_{\eta}\right)_{\xi}+\left(z_{\zeta} x_{\xi}-z_{\xi} x_{\zeta}\right)_{\eta}+\left(z_{\xi} x_{\eta}-z_{\eta} x_{\xi}\right)_{\zeta}=0 \\
I_{z}= & \left(x_{\eta} y_{\zeta}-x_{\zeta} y_{\eta}\right)_{\xi}+\left(x_{\zeta} y_{\xi}-x_{\xi} y_{\zeta}\right)_{\eta}+\left(x_{\xi} y_{\eta}-x_{\eta} y_{\xi}\right)_{\zeta}=0, \\
I_{t}= & -\left(x_{\tau} \tilde{\xi}_{x}+y_{\tau} \tilde{\xi}_{y}+z_{\tau} \tilde{\xi}_{z}\right)_{\xi}-\left(x_{\tau} \tilde{\eta}_{x}+y_{\tau} \tilde{\eta}_{y}+z_{\tau} \tilde{\eta}_{z}\right)_{\eta}-\left(x_{\tau} \tilde{\zeta}_{x}+y_{\tau} \tilde{\zeta}_{y}+z_{\tau} \tilde{\zeta}_{z}\right)_{\zeta} \\
& +\left[x_{\xi}\left(y_{\eta} z_{\zeta}-y_{\zeta} z_{\eta}\right)-x_{\eta}\left(y_{\xi} z_{\zeta}-y_{\zeta} z_{\xi}\right)+x_{\zeta}\left(y_{\xi} z_{\eta}-y_{\eta} z_{\xi}\right)\right]_{\tau}=0 .
\end{aligned}\right.
$$

Then

$$
\frac{\partial Q}{\partial \tau}=0
$$

that is, the uniform flow conditions are held. In a finite difference discretization, all four of the identities $I_{x}$ to $I_{t}$ in equation (16) must hold numerically to achieve the free-stream preserving condition. For stationary meshes, only the first three identities for $I_{x}, I_{y}$ and $I_{z}$ are required.

3.2. The numerical scheme. We will construct numerical fluxes $\hat{E}_{i+1 / 2, j, k}$, $\hat{F}_{i, j+1 / 2, k}$ and $\hat{G}_{i, j, k+1 / 2}$ such that

$$
\begin{aligned}
& \frac{\hat{E}_{i+1 / 2, j, k}-\hat{E}_{i-1 / 2, j, k}}{\Delta \xi}=\left.(\tilde{E})_{\xi}\right|_{\left(\xi_{i}, \eta_{j}, \zeta_{k}\right)}+O\left(\Delta \xi^{k}\right), \\
& \frac{\hat{F}_{i, j+1 / 2, k}-\hat{F}_{i, j-1 / 2, k}}{\Delta \eta}=\left.(\tilde{F})_{\eta}\right|_{\left(\xi_{i}, \eta_{j}, \zeta_{k}\right)}+O\left(\Delta \eta^{k}\right), \\
& \frac{\hat{G}_{i, j, k+1 / 2}-\hat{G}_{i, j, k-1 / 2}}{\Delta \zeta}=\left.(\tilde{G})_{\zeta}\right|_{\left(\xi_{i}, \eta_{j}, \zeta_{k}\right)}+O\left(\Delta \zeta^{k}\right) .
\end{aligned}
$$

The numerical flux $\hat{E}_{i+1 / 2, j, k}$ is obtained by the one dimensional WENO approximation procedure described in Section 2, with $Q(\xi)=Q\left(\xi, \eta_{j}, \zeta_{k}\right)$ and with $j, k$ fixed. We summarize the procedure in the following:

1. Get $Q_{i+1 / 2, j, k}^{ \pm}$through WENO interpolation on $Q$ in curvilinear coordinates $(\xi, \eta, \zeta, \tau)$ in the $\xi$-direction for fixed $j$ and $k$. Here the superscripts \pm refer to one-point left/right biased stencils. 
2. Construct the first term $h\left(Q_{i+1 / 2, j, k}^{-}, Q_{i+1 / 2, j, k}^{+}\right)$. For the metrics $\tilde{\xi}_{x}, \tilde{\xi}_{y}, \tilde{\xi}_{z}$ and $\tilde{\xi}_{t}$ at the half point $\left(\xi_{i+1 / 2}, \eta_{j}, \zeta_{k}\right)$, we obtain them using fixed-stencil interpolation approximation

$$
\left.\tilde{\xi}_{\gamma}\right|_{i+1 / 2, j, k}=\left.\sum_{l=-p_{1}}^{q_{1}} a_{l} \tilde{\xi}_{\gamma}\right|_{(i+l, j, k)}+O\left(\Delta \xi^{p_{1}+q_{1}+1}\right),
$$

where $p_{1}+q_{1}=k, \gamma$ stands for $x, y, z$, or $t$ and $a_{l}$ are constants not dependent on $\Delta \xi$. For instance, when $k=5$, we use central approximation and have

$$
\begin{aligned}
\left.\tilde{\xi}_{\gamma}\right|_{i+1 / 2, j, k} \approx & \frac{1}{256}\left(\left.3 \tilde{\xi}_{\gamma}\right|_{i-2, j, k}-\left.25 \tilde{\xi}_{\gamma}\right|_{i-1, j, k}+\left.150 \tilde{\xi}_{\gamma}\right|_{i, j, k}+\left.150 \tilde{\xi}_{\gamma}\right|_{i+1, j, k}\right. \\
& \left.-\left.25 \tilde{\xi}_{\gamma}\right|_{i+2, j, k}+\left.3 \tilde{\xi}_{\gamma}\right|_{i+3, j, k}\right) .
\end{aligned}
$$

3. For the term $\partial^{2} \tilde{E} / \partial \xi^{2}$, since there is an extra $\Delta \xi^{2}$ as a coefficient, interpolate only with $(k-2)$-th order accuracy

$$
\left.\Delta \xi^{2} \frac{\partial^{2} \tilde{E}}{\partial \xi^{2}}\right|_{i+1 / 2, j, k}=\left.\sum_{l=-p_{1}}^{q_{1}} b_{l} \tilde{E}\right|_{(i+l, j, k)}+O\left(\Delta \xi^{p_{1}+q_{1}+1}\right),
$$

where $p_{1}+q_{1}=k$ and $b_{l}$ are constants independent of $\Delta \xi$. When $k=5$, the following central approximation can be used

$$
\begin{aligned}
\left.\Delta \xi^{2} \frac{\partial^{2} \tilde{E}}{\partial \xi^{2}}\right|_{i+1 / 2, j, k} \approx & \frac{1}{48}\left(-\left.5 \tilde{E}\right|_{i-2, j, k}+\left.39 \tilde{E}\right|_{i-1, j, k}-\left.34 \tilde{E}\right|_{i, j, k}-\left.34 \tilde{E}\right|_{i+1, j, k}\right. \\
& \left.+\left.39 \tilde{E}\right|_{i+2, j, k}-\left.5 \tilde{E}\right|_{i+3, j, k}\right) .
\end{aligned}
$$

4. Approximate the remaining terms similarly as in step 3. For example,

$$
\left.\Delta \xi^{4} \frac{\partial^{4} \tilde{E}}{\partial \xi^{4}}\right|_{i+1 / 2, j, k}=\left.\sum_{l=-p_{1}}^{q_{1}} c_{l} \tilde{E}\right|_{(i+l, j, k)}+O\left(\Delta \xi^{p_{1}+q_{1}+1}\right)
$$

where $p_{1}+q_{1}=k$ and $c_{l}$ are constants independent of $\Delta \xi$. When $k=5$, the following central approximation can be used

$$
\begin{aligned}
\left.\Delta \xi^{4} \frac{\partial^{4} \tilde{E}}{\partial \xi^{4}}\right|_{i+1 / 2, j, k} \approx & \frac{1}{2}\left(\left.\tilde{E}\right|_{i-2, j, k}-\left.3 \tilde{E}\right|_{i-1, j, k}+\left.2 \tilde{E}\right|_{i, j, k}+\left.2 \tilde{E}\right|_{i+1, j, k}\right. \\
& \left.-\left.3 \tilde{E}\right|_{i+2, j, k}+\left.\tilde{E}\right|_{i+3, j, k}\right)
\end{aligned}
$$

5. Form the numerical flux as the sum of all the terms above

$$
\begin{aligned}
\hat{E}_{i+1 / 2, j, k}= & h\left(Q_{i+1 / 2, j, k}^{-}, Q_{i+1 / 2, j, k}^{+}\right)-\left.\frac{1}{24} \Delta \xi^{2} \frac{\partial^{2} \tilde{E}}{\partial \xi^{2}}\right|_{i+1 / 2, j, k} \\
& +\left.\frac{7}{5760} \Delta \xi^{4} \frac{\partial^{4} \tilde{E}}{\partial \xi^{4}}\right|_{i+1 / 2, j, k}+\ldots
\end{aligned}
$$

Likewise, the numerical fluxes $\hat{F}_{i, j+1 / 2, k}$ and $\hat{G}_{i, j, k+1 / 2}$ are obtained by the one dimensional WENO approximation procedure, with $Q(\eta)=Q\left(\xi_{i}, \eta, \zeta_{k}\right)$ and with $i, k$ fixed, or with $Q(\zeta)=Q\left(\xi_{i}, \eta_{j}, \zeta\right)$ and with $i, j$ fixed. 
Notice that $Q_{i+1 / 2, j, k}^{ \pm}=Q_{\ell, j, k}=Q$ if $Q$ has the same value at all grid points. Thus we have

$$
\begin{aligned}
h\left(Q_{i+1 / 2, j, k}^{-}, Q_{i+1 / 2, j, k}^{+}\right) & =h(Q, Q)=\left.\tilde{E}(Q)\right|_{i+1 / 2, j, k} \\
& =\left.\left(\tilde{\xi}_{t} Q+\tilde{\xi}_{x} E(Q)+\tilde{\xi}_{y} F(Q)+\tilde{\xi}_{z} G(Q)\right)\right|_{i+1 / 2, j, k} .
\end{aligned}
$$

Since $E=E(Q), F=F(Q)$ and $G=G(Q)$ are also constants, we have

$$
\begin{aligned}
h\left(Q_{i+1 / 2, j, k}^{-}, Q_{i+1 / 2, j, k}^{+}\right) & =\left.\sum_{l=-p_{1}}^{q_{1}} a_{l}\left(\tilde{\xi}_{t} Q+\tilde{\xi}_{x} E+\tilde{\xi}_{y} F+\tilde{\xi}_{z} G\right)\right|_{i+l, j, k}, \\
\left.\Delta \xi^{2} \frac{\partial^{2} \tilde{E}}{\partial \xi^{2}}\right|_{i+1 / 2, j, k} & =\left.\sum_{l=-p_{1}}^{q_{1}} b_{l} \tilde{E}\right|_{(i+l, j, k)} \\
& =\left.\sum_{l=-p_{1}}^{q_{1}} b_{l}\left(\tilde{\xi}_{t} Q+\tilde{\xi}_{x} E+\tilde{\xi}_{y} F+\tilde{\xi}_{z} G\right)\right|_{i+l, j, k}, \\
\left.\Delta \xi^{4} \frac{\partial^{4} \tilde{E}}{\partial \xi^{4}}\right|_{i+1 / 2, j, k} & =\sum_{l=-p_{1}}^{q_{1}} c_{l} \tilde{E}_{(i+l, j, k)} \\
& =\left.\sum_{l=-p_{1}}^{q_{1}} c_{l}\left(\tilde{\xi}_{t} Q+\tilde{\xi}_{x} E+\tilde{\xi}_{y} F+\tilde{\xi}_{z} G\right)\right|_{i+l, j, k} .
\end{aligned}
$$

Thus, $\hat{E}_{i+1 / 2, j, k}$ has the form

$$
\begin{aligned}
\hat{E}_{i+1 / 2, j, k} & =\left.Q \sum_{l=-p_{1}}^{q_{1}} d_{l} \tilde{\xi}_{t}\right|_{i+l, j, k}+\left.E \sum_{l=-p_{1}}^{q_{1}} d_{l} \tilde{\xi}_{x}\right|_{i+l, j, k} \\
& +\left.F \sum_{l=-p_{1}}^{q_{1}} d_{l} \tilde{\xi}_{y}\right|_{i+l, j, k}+\left.G \sum_{l=-p_{1}}^{q_{1}} d_{l} \tilde{\xi}_{z}\right|_{i+l, j, k},
\end{aligned}
$$

where $d_{l}$ are constants independent of $\Delta \xi$. We therefore have the flux difference

$$
\begin{aligned}
\frac{\hat{E}_{i+1 / 2, j, k}-\hat{E}_{i-1 / 2, j, k}}{\Delta \xi} & =\left.Q \frac{1}{\Delta \xi} \sum_{l=-p_{1}-1}^{q_{1}} e_{l} \tilde{\xi}_{t}\right|_{i+l, j, k}+\left.E \frac{1}{\Delta \xi} \sum_{l=-p_{1}-1}^{q_{1}} e_{l} \tilde{\xi}_{x}\right|_{i+l, j, k} \\
& +\left.F \frac{1}{\Delta \xi} \sum_{l=-p_{1}-1}^{q_{1}} e_{l} \tilde{\xi}_{y}\right|_{i+l, j, k}+\left.G \frac{1}{\Delta \xi} \sum_{l=-p_{1}-1}^{q_{1}} e_{l} \tilde{\xi}_{z}\right|_{i+l, j, k},
\end{aligned}
$$

where the $e_{l}$ are constants not dependent on $\Delta \xi$. We can see that, when $Q$ are constants, our scheme degenerates to a linear scheme. Since $(\tilde{E})_{\xi}=Q\left(\tilde{\xi}_{t}\right)_{\xi}+E\left(\tilde{\xi}_{x}\right)_{\xi}+$ $F\left(\tilde{\xi}_{y}\right)_{\xi}+G\left(\tilde{\xi}_{z}\right)_{\xi}$, we can get the approximation

$$
\left(\tilde{\xi}_{\gamma}\right)_{\xi}=\left.\frac{1}{\Delta \xi} \sum_{l=-p_{1}-1}^{q_{1}} e_{l} \tilde{\xi}_{\gamma}\right|_{i+l, j, k},
$$

where $\gamma$ stands for $x, y, z$ or $t$. 
Similarity, in the $\eta$ - and $\zeta$-directions, we also have

$$
\begin{aligned}
\begin{aligned}
\frac{\hat{F}_{i, j+1 / 2, k}-\hat{F}_{i, j-1 / 2, k}}{\Delta \eta} & =\left.Q \frac{1}{\Delta \eta} \sum_{m=-p_{2}-1}^{q_{2}} f_{m} \tilde{\eta}_{t}\right|_{i, j+m, k}+\left.E \frac{1}{\Delta \eta} \sum_{m=-p_{2}-1}^{q_{2}} f_{m} \tilde{\eta}_{x}\right|_{i, j+m, k} \\
& +\left.F \frac{1}{\Delta \eta} \sum_{m=-p_{2}-1}^{q_{2}} f_{m} \tilde{\eta}_{y}\right|_{i, j+m, k}+\left.G \frac{1}{\Delta \eta} \sum_{m=-p_{2}-1}^{q_{2}} f_{m} \tilde{\eta}_{z}\right|_{i, j+m, k} \\
\frac{\hat{G}_{i, j, k+1 / 2}-\hat{G}_{i, j, k-1 / 2}}{\Delta \zeta} & =\left.Q \frac{1}{\Delta \zeta} \sum_{n=-p_{3}-1}^{q_{3}} g_{n} \tilde{\zeta}_{t}\right|_{i, j, k+n}+\left.E \frac{1}{\Delta \zeta} \sum_{n=-p_{3}-1}^{q_{3}} g_{n} \tilde{\zeta}_{x}\right|_{i, j, k+n} \\
& +\left.F \frac{1}{\Delta \zeta} \sum_{n=-p_{3}-1}^{q_{3}} g_{n} \tilde{\zeta}_{y}\right|_{i, j, k+n}+\left.G \frac{1}{\Delta \zeta} \sum_{n=-p_{3}-1}^{q_{3}} g_{n} \tilde{\zeta}_{z}\right|_{i, j, k+n}
\end{aligned}
\end{aligned}
$$

where $f_{m}$ and $g_{n}$ are all constants.

In the first step, $\tilde{Q}$, instead of $Q$, could also be used as the interpolation quantity for achieving the formal order of accuracy. However, since $\tilde{Q}$ includes the Jacobian, the weights evaluated in the weighted averaging procedure will be adversely affected, even under the free-stream condition. For this reason, $\tilde{Q}$ values are not used for the WENO interpolation procedure in our algorithm formulation. The validity of using $Q$ in our procedure is shown later.

3.3. SCL and VCL. For finite difference numerical methods, Thomas and Lombard [11] gave the following "conservation" metric expressions instead of equation (11)

$$
\left\{\begin{array}{lll}
\tilde{\xi}_{x}=\left(y_{\eta} z\right)_{\zeta}-\left(y_{\zeta} z\right)_{\eta}, & \tilde{\xi}_{y}=\left(z_{\eta} x\right)_{\zeta}-\left(z_{\zeta} x\right)_{\eta}, & \tilde{\xi}_{z}=\left(x_{\eta} y\right)_{\zeta}-\left(x_{\zeta} y\right)_{\eta} \\
\tilde{\eta}_{x}=\left(y_{\zeta} z\right)_{\xi}-\left(y_{\xi} z\right)_{\zeta}, & \tilde{\eta}_{y}=\left(z_{\zeta} x\right)_{\xi}-\left(z_{\xi} x\right)_{\zeta}, & \tilde{\eta}_{z}=\left(x_{\zeta} y\right)_{\xi}-\left(x_{\xi} y\right)_{\zeta} \\
\tilde{\zeta}_{x}=\left(y_{\xi} z\right)_{\eta}-\left(y_{\eta} z\right)_{\xi}, & \tilde{\zeta}_{y}=\left(z_{\xi} x\right)_{\eta}-\left(z_{\eta} x\right)_{\xi}, & \tilde{\zeta}_{z}=\left(x_{\xi} y\right)_{\eta}-\left(x_{\eta} y\right)_{\xi}
\end{array}\right.
$$

Equations (11) and (26) are equivalent mathematically but not necessarily numerically. Visbal and Gaitonde [13] reported that a compact difference scheme, which is applied to the generalized coordinate system, can preserve the free-stream condition if the conservation form (26) of the metric terms is evaluated using the same formulas as those used in the evaluation of the conservation terms, at least for free-stream solutions. Here we use the same idea in our method. For example,

$$
\begin{aligned}
\left.\tilde{\xi}_{x}\right|_{i, j, k} & =\left.\frac{1}{\Delta \zeta} \sum_{n=-p_{3}-1}^{q_{3}} g_{n}\left(y_{\eta} z\right)\right|_{i, j, k+n}-\left.\frac{1}{\Delta \eta} \sum_{m=-p_{2}-1}^{q_{2}} f_{m}\left(y_{\zeta} z\right)\right|_{i, j+m, k} \\
& =\left.\left.\frac{1}{\Delta \zeta \Delta \eta} \sum_{n=-p_{3}-1}^{q_{3}} g_{n} z\right|_{i, j, k+n} \sum_{m=-p_{2}-1}^{q_{2}} f_{m} y\right|_{i, j+m, k+n} \\
& -\left.\left.\frac{1}{\Delta \eta \Delta \zeta} \sum_{m=-p_{2}-1}^{q_{2}} f_{m} z\right|_{i, j+m, k} \sum_{n=-p_{3}-1}^{q_{3}} g_{n} y\right|_{i, j+m, k+n} .
\end{aligned}
$$


Thus

$$
\begin{aligned}
I_{x} & =\left(\tilde{\xi}_{x}\right)_{\xi}+\left(\tilde{\eta}_{x}\right)_{\eta}+\left(\tilde{\zeta}_{x}\right)_{\zeta} \\
& =\left.\frac{1}{\Delta \xi} \sum_{l=-p_{1}-1}^{q_{1}} e_{l} \tilde{\xi}_{x}\right|_{i+l, j, k}+\left.\frac{1}{\Delta \eta} \sum_{m=-p_{2}-1}^{q_{2}} f_{m} \tilde{\eta}_{x}\right|_{i, j+m, k}+\left.\frac{1}{\Delta \zeta} \sum_{n=-p_{3}-1}^{q_{3}} g_{n} \tilde{\zeta}_{x}\right|_{i, j, k+n} \\
& =\left.\left.\frac{1}{\Delta \xi \Delta \zeta \eta \eta} \sum_{l=-p_{1}-1}^{q_{1}} e_{l} \sum_{n=-p_{3}-1}^{q_{3}} g_{n} z\right|_{i+l, j, k+n} \sum_{m=-p_{2}-1}^{q_{2}} f_{m} y\right|_{i+l, j+m, k+n} \\
& -\left.\left.\frac{1}{\Delta \xi \Delta \eta \zeta} \sum_{l=-p_{1}-1}^{q_{1}} e_{l} \sum_{m=-p_{2}-1}^{q_{2}} f_{m} z\right|_{i+l, j+m, k} \sum_{n=-p_{3}-1}^{q_{3}} g_{n} y\right|_{i+l, j+m, k+n} \\
& +\left.\left.\frac{1}{\Delta \eta \Delta \xi \Delta \zeta} \sum_{m=-p_{2}-1}^{q_{2}} f_{m} \sum_{l=-p_{1}-1}^{q_{1}} e_{l} z\right|_{i+l, j+m, k} \sum_{n=-p_{3}-1}^{q_{3}} g_{n} y\right|_{i+l, j+m, k+n} \\
& -\left.\left.\frac{1}{\Delta \eta \Delta \zeta \Delta \xi} \sum_{m=-p_{2}-1}^{q_{2}} f_{m} \sum_{n=-p_{3}-1}^{q_{3}} g_{n} z\right|_{i, j+m, k+n} \sum_{l=-p_{1}-1}^{q_{1}} e_{l} y\right|_{i+l, j+m, k+n} \\
& +\left.\left.\frac{1}{\Delta \zeta \Delta \eta \Delta \xi} \sum_{n=-p_{3}-1}^{q_{3}} g_{n} \sum_{m=-p_{2}-1}^{q_{2}} f_{m} z\right|_{i, j+m, k+n} \sum_{l=-p_{1}-1}^{q_{3}} e_{l} y\right|_{i+l, j+m, k+n} \\
& -\left.\frac{1}{\Delta \zeta \Delta \xi \Delta \eta} \sum_{n=-p_{3}-1}^{q_{3}} g_{n} \sum_{l=-p_{1}-1}^{q_{1}} e_{l} z\right|_{i+l, j, k+n} \sum_{m=-p_{2}-1}^{\left.f_{m} y\right|_{i+l, j+m, k+n}} \\
& =0 .
\end{aligned}
$$

Similarly, we also have

$$
I_{y}=0, \quad I_{z}=0 .
$$

Thus, we can get the SCL.

For moving meshes, the VCL identity $I_{t}=0$ must also be satisfied. We simply invoke the VCL identity to evaluate $(1 / J)_{\tau}$, i.e.

$$
(1 / J)_{\tau}=-\left[\left(\tilde{\xi}_{t}\right)_{\xi}+\left(\tilde{\eta}_{t}\right)_{\eta}+\left(\tilde{\zeta}_{t}\right)_{\zeta}\right]
$$

For the standard conservative finite difference WENO methods [4] in the generalized coordinates, the idea of Visbal and Gaitonde [13] cannot be applied, since it requires the same scheme for the evaluation of the metric and the convection terms. The Jacobian and metrics cannot be evaluated by the upwinding procedure of WENO because they cannot be split to the nonlinear upwind components. Thus, compact difference formulas are used to evaluate the metric terms in [7].

4. Numerical results. In this section, we will discuss the results of both stationary and dynamical meshes. We denote S-LF and S-R as the standard finite difference WENO scheme of Jiang and Shu [4] with the Lax-Friedrichs flux splitting and the Roe splitting respectively. A-LF and A-HLLC are used to denote the methods under consideration with the alternative flux formulation and with the Lax-Friedrichs flux and the HLLC flux respectively. The third-order TVD Runge-Kutta scheme is 
used for time integration

$$
\begin{aligned}
& u^{(1)}=u^{n}+\Delta t L\left(u^{n}\right), \\
& u^{(2)}=\frac{3}{4} u^{n}+\frac{1}{4} u^{(1)}+\frac{1}{4} \Delta t L\left(u^{(1)}\right), \\
& u^{n+1}=\frac{1}{3} u^{n}+\frac{2}{3} u^{(2)}+\frac{2}{3} \Delta t L\left(u^{(2)}\right) .
\end{aligned}
$$

The specific heat ratio of the fluid in the test cases is set as $\gamma=1.4$. Compact difference formulas, as described in [7], are used to evaluate the metric terms for the standard finite difference WENO scheme.

4.1. Free-stream preserving properties. The fluid in the test cases is nondimensionalized by the density and the speed of sound for the free-stream condition. In this test, an $x$-direction free-stream of Mach number 0.5 is imposed. Thus the $y$-direction velocity $v$ and the $z$-direction velocity $w$ are expected to remain machine zero.

EXAMPLE 1. Firstly, we will test the free-stream preservation property on a stationary wavy grid, which is expressed as following:

$$
\begin{aligned}
& x_{i, j, k}=x_{\min }+\Delta x_{0}\left[(i-1)+A_{x} \sin \frac{n_{x y} \pi(j-1) \Delta y_{0}}{L_{y}} \sin \frac{n_{x z} \pi(k-1) \Delta z_{0}}{L_{z}}\right], \\
& y_{i, j, k}=y_{\min }+\Delta y_{0}\left[(j-1)+A_{y} \sin \frac{n_{y z} \pi(k-1) \Delta z_{0}}{L_{z}} \sin \frac{n_{y x} \pi(i-1) \Delta x_{0}}{L_{x}}\right], \\
& z_{i, j, k}=z_{\min }+\Delta z_{0}\left[(k-1)+A_{z} \sin \frac{n_{z x} \pi(i-1) \Delta x_{0}}{L_{x}} \sin \frac{n_{z y} \pi(j-1) \Delta y_{0}}{L_{y}}\right],
\end{aligned}
$$

where

$$
\begin{array}{lll}
i=1,2, \cdots, I_{\max }, & j=1,2, \cdots, J_{\max }, & k=1,2, \cdots, K_{\max }, \\
\Delta x_{0}=\frac{L_{x}}{I_{\max }-1}, & \Delta y_{0}=\frac{L_{y}}{J_{\max }-1}, & \Delta z_{0}=\frac{L_{z}}{K_{\max }-1}, \\
x_{\min }=-\frac{L_{x}}{2}, & y_{\min }=-\frac{L_{y}}{2}, & z_{\min }=-\frac{L_{z}}{2} .
\end{array}
$$

The wavy grid parameters used in this test are $I_{\max }=J_{\max }=K_{\max }=21, L_{x}=L_{y}=$ $L_{z}=4, A_{x}=A_{y}=A_{z}=1$, and $n_{x y}=n_{x z}=\ldots=4$, and the grid is shown in Fig. 1 . Time step is taken as $\Delta t=0.05$, and flow fields at $t=10$ are examined. We list the $L_{2}$ errors of $v$ and $w$ in Table 1 . We can see that A-LF and A-HLLC have errors less than $10^{-14}$, which are close to the machine zero. However, the S-LF and S-R schemes have large errors on the level of $10^{-3}$. These demonstrate that our schemes with the alternative flux formulation are appropriate for free-stream preservation, while neither S-LF nor S-R could preserve free-stream solutions.

TABLE 1

$L_{2}$ errors of $v$ and $w$ components in the free-stream preservation test on a wavy grid.

\begin{tabular}{|c|c|c|}
\hline & $v$-component & $w$-component \\
\hline S-LF & $9.33 \mathrm{E}-03$ & $9.32 \mathrm{E}-03$ \\
S-R & $9.34 \mathrm{E}-03$ & $9.18 \mathrm{E}-03$ \\
A-LF & $2.03 \mathrm{E}-15$ & $1.54 \mathrm{E}-15$ \\
A-HLLC & $4.05 \mathrm{E}-15$ & $2.67 \mathrm{E}-15$ \\
\hline
\end{tabular}




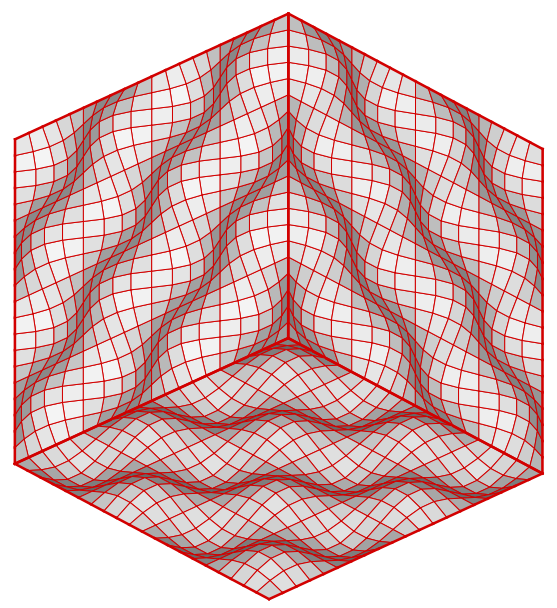

FIG. 1. A stationary wavy grid for the free-stream preserving test.

EXAMPLE 2. Next, we will test the free-stream preservation on a randomized grid. Uniform grids constructed for the mesh $\Delta x=\Delta y=\Delta z=0.2$ with $21 \times 21 \times 21$ grid points are randomized with $20 \%$ magnitude grid spacing in a random direction, and the mesh is shown in Fig. 2. The same time step $\Delta t=0.05$ is used. We also test our results at $t=10$. Results for $L_{2}$ errors of $v$ and $w$ are shown in Table 2. Similar to our observations in Example 1, the S-LF and S-R schemes both have large errors, while A-LF and A-HLLC have errors close to machine zero. So, on a randomized grid, A-LF and A-HLLC can also preserve free-stream solutions.

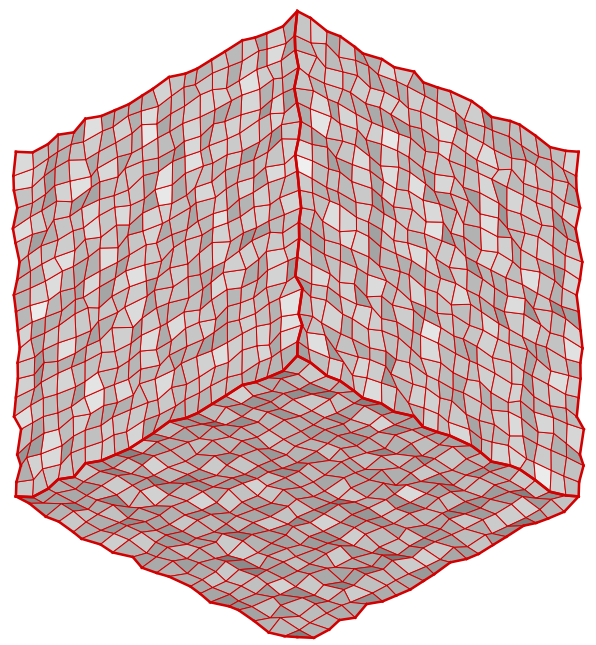

FIG. 2. A stationary randomized grid for the free-stream preserving test.

EXAMPLE 3. In this example, we consider the free-stream condition on the spherical grid shown in Fig. 3. The computational domain is chosen as $[2,4] \times[0,2] \times[-2,0]$ 
TABLE 2

$L_{2}$ errors of $v$ and $w$ components in the free-stream preservation test on a randomized grid.

\begin{tabular}{|c|c|c|}
\hline & $v$-component & $w$-component \\
\hline S-LF & $7.70 \mathrm{E}-02$ & $7.53 \mathrm{E}-02$ \\
S-R & $1.21 \mathrm{E}-01$ & $1.18 \mathrm{E}-01$ \\
A-LF & $1.76 \mathrm{E}-15$ & $1.61 \mathrm{E}-15$ \\
A-HLLC & $3.16 \mathrm{E}-15$ & $2.92 \mathrm{E}-15$ \\
\hline
\end{tabular}

on the $r-\theta-\phi$ coordinate systems:

$$
\begin{aligned}
& x=r \cos (\pi \theta / 6) \cos (\pi \phi / 6), \\
& y=r \sin (\pi \theta / 6) \cos (\pi \phi / 6), \\
& z=r \sin (\pi \phi / 6) .
\end{aligned}
$$

A uniform mesh with $21 \times 21 \times 21$ grid points is used on the computational domain. The time step was taken to be $\Delta t=0.02$, and the results were again tested at the final time of $t=10 . L_{2}$ errors of $v$ and $w$ are shown in Table 3 . We can see that the numerical results for our schemes with the alternative formulation can reach machine zero, and the free-stream condition is preserved. On the other hand, both S-LF and $\mathrm{S}-\mathrm{R}$ schemes have large errors, and fail to preserve the free-stream condition.

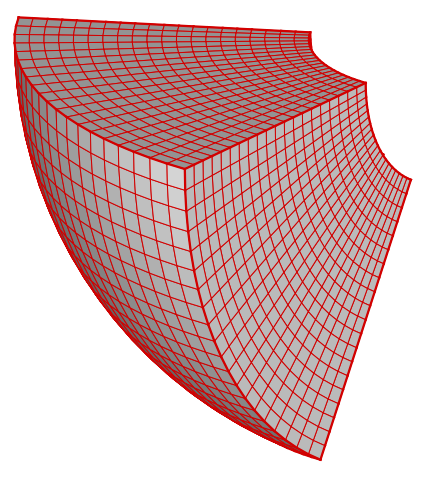

FIG. 3. A stationary spherical grid for the free-stream preserving test.

TABLE 3

$L_{2}$ errors of $v$ and $w$ components in the free-stream preservation test on a spherical grid.

\begin{tabular}{|c|c|c|}
\hline & $v$-component & $w$-component \\
\hline S-LF & $2.80 \mathrm{E}-07$ & $2.67 \mathrm{E}-07$ \\
S-R & $1.34 \mathrm{E}-06$ & $6.79 \mathrm{E}-07$ \\
A-LF & $5.92 \mathrm{E}-16$ & $5.42 \mathrm{E}-16$ \\
A-HLLC & $1.11 \mathrm{E}-15$ & $1.09 \mathrm{E}-15$ \\
\hline
\end{tabular}


EXAMPLE 4. Finally, we will test the free-stream preservation on a moving wavy mesh:

$$
\begin{aligned}
& x_{i, j, k}(\tau)=x_{\min }+\Delta x_{0}\left[(i-1)+A_{x} \sin (2 \pi \omega \tau) \sin \frac{n_{x y} \pi(j-1) \Delta y_{0}}{L_{y}} \sin \frac{n_{x z} \pi(k-1) \Delta z_{0}}{L_{z}}\right], \\
& y_{i, j, k}(\tau)=y_{\min }+\Delta y_{0}\left[(j-1)+A_{y} \sin (2 \pi \omega \tau) \sin \frac{n_{y z} \pi(k-1) \Delta z_{0}}{L_{z}} \sin \frac{n_{y x} \pi(i-1) \Delta x_{0}}{L_{x}}\right], \\
& z_{i, j, k}(\tau)=z_{\min }+\Delta z_{0}\left[(k-1)+A_{z} \sin (2 \pi \omega \tau) \sin \frac{n_{z x} \pi(i-1) \Delta x_{0}}{L_{x}} \sin \frac{n_{z y} \pi(j-1) \Delta y_{0}}{L_{y}}\right],
\end{aligned}
$$

where

$$
\begin{array}{lll}
i=1,2, \cdots, I_{\max }, & j=1,2, \cdots, J_{\max }, & k=1,2, \cdots, K_{\max }, \\
\Delta x_{0}=\frac{L_{x}}{I_{\max }-1}, & \Delta y_{0}=\frac{L_{y}}{J_{\max _{2}-1}}, & \Delta z_{0}=\frac{L_{z}}{K_{\max }-1}, \\
x_{\min }=-\frac{L_{x}}{2}, & y_{\min }=-\frac{L_{y}}{2}, & z_{\min }=-\frac{L_{z}}{2},
\end{array}
$$

with the specified parameters $I_{\max }=J_{\max }=K_{\max }=31, L_{x}=L_{y}=L_{z}=12$, $A_{x}=A_{y}=A_{z}=1.5$, and $n_{x y}=n_{x z}=\ldots=4$, and the frequency of oscillation $\omega=1.0$. We choose the time step $\Delta t=0.001$, and the flow fields at $t=0.25$ are examined. Fig. 4 shows the mesh at the final time, and numerical results are listed in Table 4. Similar to the stationary meshes, A-LF and A-HLLC can preserve the free-stream condition, while S-LF and S-R cannot.

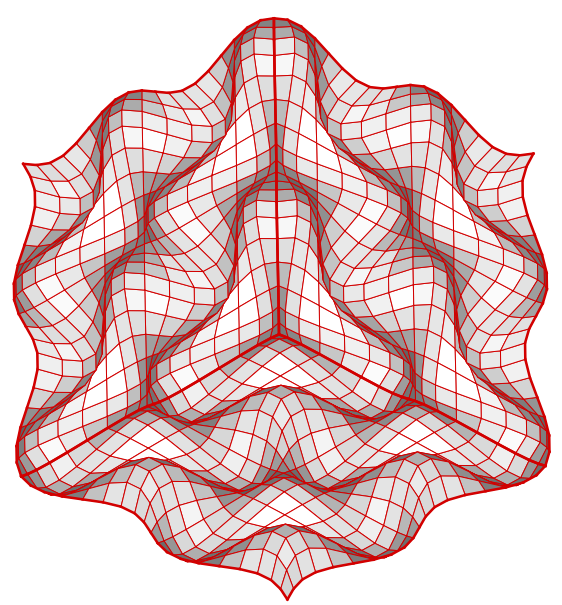

FIG. 4. A moving wavy grid for the free-stream preserving test at $t=0.25$.

4.2. Vortex preservation properties. Here, two-dimensional moving vortex problems are used to examine vortex preservation properties. The fluid is nondimensionalized by the density and the speed of the sound for the free-stream condition, as in the free-stream preservation test. An isentropic vortex whose center is located at $\left(x_{c}, y_{c}\right)=(0,0)$ is set on the free-stream of Mach number 0.5 as the initial condition, where the velocity, temperature, and entropy fluctuations of an isentropic vortex are 
TABLE 4

$L_{2}$ errors of $v$ and $w$ components in the free-stream preservation test on a moving wavy grid.

\begin{tabular}{|c|c|c|}
\hline & $v$-component & $w$-component \\
\hline S-LF & $4.45 \mathrm{E}-02$ & $4.45 \mathrm{E}-02$ \\
S-R & $4.46 \mathrm{E}-02$ & $4.46 \mathrm{E}-02$ \\
A-LF & $6.91 \mathrm{E}-16$ & $6.98 \mathrm{E}-16$ \\
A-HLLC & $3.71 \mathrm{E}-16$ & $3.72 \mathrm{E}-16$ \\
\hline
\end{tabular}

expressed as follows:

$$
\begin{aligned}
& (\delta u, \delta v)=\varepsilon \lambda e^{\alpha\left(1-\lambda^{2}\right)}(\sin \theta,-\cos \theta) \\
& \delta T=-\frac{(\gamma-1) \varepsilon^{2}}{4 \alpha \gamma} e^{2 \alpha\left(1-\lambda^{2}\right)} \\
& \delta S=0
\end{aligned}
$$

where $\lambda=r / r_{c}$ and $r=\left[\left(x-x_{c}\right)^{2}+\left(y-y_{c}\right)^{2}\right]^{1 / 2}$. Here, $r_{c}=1.0$ denotes the vortex core length, $\alpha=0.204$ denotes the parameter of the length scale of the vortex decay, and $\varepsilon=0.3$ denotes the vortex strength. $T=p / \rho$ is the temperature, and $S=p / \rho^{\gamma}$ is the entropy. The boundary conditions for $\xi$ - and $\eta$-directions are set to be periodic. Numerical results are tested at $t=32$, at which time the vortex moves back to the same position as the initial condition in the grid system defined in each of the following examples.

EXAMPLE 5. First, vortex preserving properties on uniform grids are examined. Three different computational grids are constructed for the region $-8 \leq x \leq 8$ and $-8 \leq y \leq 8$, with $41 \times 41,81 \times 81$ and $161 \times 161$ grid points. Time steps $\Delta t$ are set to $0.05,0.0125$, and 0.003125 for the $41 \times 41,81 \times 81$ and $161 \times 161$ grids, respectively. These small time steps are used to minimize the temporal error from the third-order time integration. The $L_{2}$ errors and orders of accuracy for the swirl velocity are presented in Table 5, showing that all these schemes can achieve close to the designed fifth order accuracy on uniform meshes. Fig. 5 shows the most coarse computational grid and the vorticity magnitude distributions for the initial conditions and the four schemes at $t=32$. Fig. 6 shows the swirl velocity on the $\eta$-constant line passing through the vortex center. These figures demonstrate that the vortex of all these four implementations can preserve the vortex strength and swirl velocity qualitatively compared with the exact solutions.

TABLE 5

$L_{2}$ errors in the swirl velocity of the two-dimensional moving vortex problem on the uniform grid.

\begin{tabular}{|c|cc|cc|cc|cc|}
\hline & \multicolumn{2}{|c|}{ S-LF } & \multicolumn{2}{c|}{ S-R } & \multicolumn{2}{c|}{ A-LF } & \multicolumn{2}{c|}{ A-HLLC } \\
\hline$N_{x} \times N_{y}$ & $L_{2}$ errors & order & $L_{2}$ error & order & $L_{2}$ errors & order & $L_{2}$ error & order \\
\hline $41 \times 41$ & $3.54 \mathrm{E}-03$ & - & $2.92 \mathrm{E}-03$ & - & $4.64 \mathrm{E}-03$ & - & $1.81 \mathrm{E}-03$ & - \\
$81 \times 81$ & $1.88 \mathrm{E}-04$ & 4.23 & $1.42 \mathrm{E}-04$ & 4.36 & $2.50 \mathrm{E}-04$ & 4.21 & $9.21 \mathrm{E}-05$ & 4.30 \\
$161 \times 161$ & $7.51 \mathrm{E}-06$ & 4.65 & $6.14 \mathrm{E}-06$ & 4.53 & $1.05 \mathrm{E}-05$ & 4.58 & $3.93 \mathrm{E}-06$ & 4.55 \\
\hline
\end{tabular}


EXAmPLE 6. Next, we will test the vortex preserving property on wavy grids. A two-dimensional wavy grid is formulated as follows:

$$
\begin{aligned}
& x_{i, j}=x_{\min }+\Delta x_{0}\left[(i-1)+A_{x} \sin \frac{n_{x y} \pi(j-1) \Delta y_{0}}{L_{y}}\right], \\
& y_{i, j}=y_{\min }+\Delta y_{0}\left[(j-1)+A_{y} \sin \frac{n_{y x} \pi(i-1) \Delta x_{0}}{L_{x}}\right],
\end{aligned}
$$

where

$$
\begin{array}{ll}
i=1,2, \cdots, I_{\max }, & j=1,2, \cdots, J_{\max }, \\
\Delta x_{0}=\frac{L_{x}}{I_{\max }-1}, & \Delta y_{0}=\frac{L_{y}}{J_{\max }-1}, \\
x_{\min }=-\frac{L_{x}}{2}, & y_{\min }=-\frac{L_{y}}{2} .
\end{array}
$$

The parameters for the wavy grid are $L_{x}=L_{y}=16, A_{x} \Delta x_{0}=0.4, A_{y} \Delta y_{0}=0.8$, and $n_{x y}=n_{y x}=6$. Similar to the uniform grids, three different grids, with $41 \times 41,81 \times 81$ and $161 \times 161$ grid points, are used. $\Delta t$ is set to be $1 / 30,1 / 120$ and $1 / 480$ for the $41 \times 41,81 \times 81$ and $161 \times 161$ grids respectively. The $L_{2}$ errors and orders of accuracy in the swirl velocity are presented in Table 6 . These numerical results show that all the four schemes can again achieve close to the designed fifth order accuracy. Fig. 7 shows the computational grid and the vorticity magnitude distributions for the initial conditions and the four schemes at $t=32$ on the most coarse gird. In Fig. 8, the swirl velocity on the $\eta$-constant line passing through the vortex center are plotted. These figures demonstrate that S-R, A-LF and A-HLLC can preserve the vortex strength and swirl velocity qualitatively compared with the exact solutions, while S-LF has significantly worse performance. This may be caused by the high diffusive nature of the Lax-Friedrichs flux splitting in which the wavy grid participates.

TABLE 6

$L_{2}$ errors in the swirl velocity of the two-dimensional moving vortex problem on the wavy grid.

\begin{tabular}{|c|cc|cc|cc|cc|}
\hline & \multicolumn{2}{|c|}{ S-LF } & \multicolumn{2}{c|}{ S-R } & \multicolumn{2}{c|}{ A-LF } & \multicolumn{2}{c|}{ A-HLLC } \\
\hline$N_{x} \times N_{y}$ & $L_{2}$ errors & order & $L_{2}$ error & order & $L_{2}$ errors & order & $L_{2}$ error & order \\
\hline $41 \times 41$ & $6.12 \mathrm{E}-02$ & - & $2.83 \mathrm{E}-02$ & - & $3.58 \mathrm{E}-02$ & - & $2.11 \mathrm{E}-02$ & - \\
$81 \times 81$ & $5.72 \mathrm{E}-03$ & 3.42 & $2.28 \mathrm{E}-03$ & 3.63 & $2.79 \mathrm{E}-03$ & 3.69 & $1.36 \mathrm{E}-03$ & 3.96 \\
$161 \times 161$ & $2.29 \mathrm{E}-04$ & 4.64 & $1.26 \mathrm{E}-04$ & 4.17 & $1.23 \mathrm{E}-04$ & 4.50 & $6.47 \mathrm{E}-05$ & 4.39 \\
\hline
\end{tabular}

EXAMPLE 7. Now, the vortex preserving property on the randomized grids is examined. Uniform grids constructed for the region $-8 \leq x \leq 8$ and $-8 \leq y \leq 8$ with $41 \times 41$ grid points are randomized with $20 \%$ magnitude grid spacing in a random direction. Time step $\Delta t=0.01$ and numerical solutions are shown at the final time $t=32$, at which point the vortex moves back to the same position as the initial condition. Fig. 9 shows the computational grid and vorticity magnitude distribution for the initial condition and the solutions at $t=32$. Fig. 10 shows the swirl velocity on the $\eta$-constant line approximately passing through the vortex center. Results for S-LF and S-R show large numerical errors owing to the grid distortions. This indicates that metric canceling has a strong effect on the randomized grid. The result for the S-R scheme is different from that of the strongly wavy grid. This should be due to the discontinuity in the derivatives of the randomized grid points, whereas the strongly wavy grid does not have such a discontinuity, at least analytically. We emphasize that conservative finite difference schemes are not designed for grids with 
discontinuous derivatives [4]. However, in practice low quality grids may appear and we would still like to reduce numerical artifacts when finite difference schemes are used on such grids.

EXAMPLE 8. Next, we conduct vortex preserving test on a grid with an abrupt change in grid spacing. The computational grid is $-8 \leq x \leq 8$ and $-8 \leq y \leq 8$. Abrupt changes are imposed in the streamwise spatial distribution at $x=-4\left(S_{1}\right)$ and $x=4\left(S_{2}\right)$. At the location $S_{1}$, the mesh spacing is suddenly halved from $\Delta x_{2}=0.4$ to $\Delta x_{1}=0.2$. Conversely, at $S_{2}$, the mesh is abruptly coarsened back to $\Delta x_{2}$. Periodic boundary conditions are applied in both coordinate directions. The grid is uniform in the $y$-direction with $\Delta y=\Delta x_{1} . \Delta t=0.01$, and the computation is then carried out until $t=32$. From Fig. 11 and Fig. 12, we can see that S-R, A-LF and A-HLLC can preserve the vortex, while S-LF has a large error, which may again be caused by the high diffusive nature of the Lax-Friedrichs flux splitting in which the abrupt-changing grid participates.

EXAMPLE 9. In this example, we test our problems on the mesh which exhibits a localized abrupt change in the slope of $45^{\circ}$ at $x=0 . \Delta x=0.4$ and $\Delta y=0.4$. Numerical solutions are shown at $t=32$ in Fig. 13 and Fig. 14 , and $\Delta t=0.01$. We can see that all the four schemes can preserve the vortex for the mesh with localized abrupt change.

EXAMPLE 10. Finally, we test the two-dimensional moving vortex problems on a dynamical wavy grid formulated as follows:

$$
\begin{aligned}
& x_{i, j}(\tau)=x_{\min }+\Delta x_{0}\left[(i-1)+A_{x} \sin (2 \pi \omega \tau) \sin \frac{n_{x y} \pi(j-1) \Delta y_{0}}{L_{y}}\right], \\
& y_{i, j}(\tau)=y_{\min }+\Delta y_{0}\left[(j-1)+A_{y} \sin (2 \pi \omega \tau) \sin \frac{n_{y x} \pi(i-1) \Delta x_{0}}{L_{x}}\right],
\end{aligned}
$$

where

$$
\begin{array}{ll}
i=1,2, \cdots, I_{\max }, & j=1,2, \cdots, J_{\max }, \\
\Delta x_{0}=\frac{L_{x}}{I_{\max }-1}, & \Delta y_{0}=\frac{L_{y}}{J_{\max }-1}, \\
x_{\min }=-\frac{L_{x}}{2}, & y_{\min }=-\frac{L_{y}}{2} .
\end{array}
$$

The parameters for the wavy grid are $L_{x}=L_{y}=16, A_{x} \Delta x_{0}=A_{y} \Delta y_{0}=0.6$, and $n_{x y}=n_{y x}=6$. Similar to the uniform grids, three different grids with $41 \times 41,81 \times 81$ and $161 \times 161$ grid points are used. $\Delta t$ is set to $1 / 50,1 / 200$ and $1 / 800$ for the $41 \times 41$, $81 \times 81$ and $161 \times 161$ grids respectively. $L_{2}$ errors in the swirl velocity are listed in Table 7 , and vorticity magnitude distributions and swirl velocity distributions on the line approximately passing through the vortex center at the final time $t=32$ are shown in Fig. 15 and Fig. 16. Compared with S-LF and S-R, A-LF and AHLLC have better performance, which shows that metric canceling has a strong effect on the dynamically changing grids. Similar to the stationary wavy grids, the large error and distortion of the vortex for the S-LF scheme may be caused by the high diffusive nature of the Lax-Friedrichs flux splitting in which the changing wavy grid participates.

\subsection{Flow past a cylinder.}

EXAMPLE 11. In this test, we use the schemes to simulate the supersonic flow past a cylinder. In the physical space, a cylinder of unit radius is positioned at the 
TABLE 7

$L_{2}$ errors in the swirl velocity of the two-dimensional moving vortex problem on the dynamically changing wavy grid.

\begin{tabular}{|c|cc|cc|cc|cc|}
\hline & \multicolumn{2}{|c|}{ S-LF } & \multicolumn{2}{c|}{ S-R } & \multicolumn{2}{c|}{ A-LF } & \multicolumn{2}{c|}{ A-HLLC } \\
\hline$N_{x} \times N_{y}$ & $L_{2}$ errors & order & $L_{2}$ error & order & $L_{2}$ errors & order & $L_{2}$ error & order \\
\hline $41 \times 41$ & $6.16 \mathrm{E}-02$ & - & $4.04 \mathrm{E}-02$ & - & $3.08 \mathrm{E}-02$ & - & $1.66 \mathrm{E}-02$ & - \\
$81 \times 81$ & $5.77 \mathrm{E}-03$ & 3.42 & $3.70 \mathrm{E}-03$ & 3.45 & $1.91 \mathrm{E}-03$ & 4.01 & $9.45 \mathrm{E}-04$ & 4.13 \\
$161 \times 161$ & $3.15 \mathrm{E}-04$ & 4.19 & $2.40 \mathrm{E}-04$ & 3.95 & $7.93 \mathrm{E}-05$ & 4.59 & $4.12 \mathrm{E}-05$ & 4.52 \\
\hline
\end{tabular}

origin on the $x-y$ plane. The computational domain is chosen to be $[0,1] \times[0,1]$ on the $\xi-\eta$ plane. The mapping between the computational domain and the physical domain is

$$
\begin{aligned}
& x=\left(R_{x}-\left(R_{x}-1\right) \xi\right) \cos (\theta(2 \eta-1)), \\
& y=\left(R_{y}-\left(R_{y}-1\right) \xi\right) \sin (\theta(2 \eta-1)) .
\end{aligned}
$$

Here we take $R_{x}=3, R_{y}=6$, and $\theta=5 \pi / 12$. A uniform mesh of $60 \times 80$ in the computational domain is used. An illustration of the mesh in the physical space is shown in Fig. 17(a), which draws every other grid line. The problem is initialized by a Mach 3 shock moving toward the cylinder from the left. The reflective boundary condition is imposed at the surface of the cylinder, i.e., $\xi=1$, the inflow boundary condition is applied at $\xi=0$, and the outflow boundary condition is applied $\eta=0,1$. From Fig. 17, we can see that all the four schemes can simulate the supersonic flow past a cylinder well.

5. Concluding remarks. In this paper, we have discussed the performance of conservative finite difference WENO schemes based on an alternative flux formulation $[10,5]$ on generalized meshes, and compared their performance preserving free-stream and vortex solution with those of the standard finite difference WENO schemes. In our scheme with the alternative flux formulation, the WENO interpolation of the solution and its derivatives are used to directly construct the numerical flux, instead of the usual practice of reconstructing the flux functions. Thus, the numerical technique of Visbal and Gaitonde [13] for free-stream preservation is applicable to the scheme with the alternative flux formulation but not to the standard finite difference WENO schemes based on the reconstruction of the flux functions.

The free-stream and vortex preservation properties for the new schemes have been investigated both theoretically and numerically. Regarding the free-stream preserving tests, it has been found that the scheme with the alternative flux formulation can preserve the free-stream condition on both stationary and dynamically changing meshes, while the standard WENO schemes cannot. Through the vortex preservation tests, we have found that the standard finite difference WENO schemes have a rather large error arising from the metric terms on randomized and moving grids, while the finite difference WENO schemes with the alternative flux formulation based both on the Lax-Friedrichs flux and on the HLLC flux can simulate the vortex well for all grids. For the problem of flow passing a cylinder, both the scheme with the alternative flux formulation and the standard WENO schemes can simulate the solution well. 


\section{REFERENCES}

[1] D. BAlsara AND C.-W. SHu, Monotonicity preserving weighted essentially non-oscillatory schemes with increasingly high order of accuracy, Journal of Computational Physics, 160 (2000), pp. 405-452.

[2] X. CAi AND F. LADEINDE, Performance of WENO scheme in generalized curvilinear coordinate systems, AIAA Paper 2008-36, 2008.

[3] E. Carlini, R. Ferretti, and G. Russo, A weighted essentially nonoscillatory, large timestep scheme for Hamilton-Jacobi equations, SIAM Journal on Scientific Computing, 27 (2005), pp. 1071-1091.

[4] G. S. Jiang AND C.-W. SHU, Efficient implementation of weighted ENO schemes, Journal of Computational Physics, 181 (1996), pp. 202-228.

[5] Y. Jiang, C.-W. Shu And M. Zhang, An alternative formulation of finite difference WENO schemes with Lax-Wendroff time discretization for conservation laws, SIAM Journal on Scientific Computing, 35 (2013), pp. A1137-A1160.

[6] X. D. Liu, S. Osher, And T. Chan, Weighted essentially non-oscillatory schemes, Journal of Computational Physics, 115 (1994), pp. 200-212.

[7] T. Nonomura, N. IIzuka, And K. FujiI, Freestream and vortex preservation properties of high-order WENO and WCNS on curvilinear grids, Computers and Fluids, 39 (2010), pp. 197-214.

[8] K. Sebastian And C.-W. Shu, Multi domain WENO finite difference method with interpolation at sub-domain interfaces, Journal of Scientific Computing, 19 (2003), pp. 405-438.

[9] C.-W. SHU, High order weighted essentially non-oscillatory schemes for convection dominated problems, SIAM Review, 51 (2009), pp. 82-126.

[10] C.-W. Shu AND S. Osher, Efficient implementation of essentially non-oscillatory shockcapturing schemes, Journal of Computational Physics, 77 (1988), pp. 439-471.

[11] P. D. Thomas And C. K. Lombard, Geometric conservation law and its application to flow computations on moving grids, AIAA Journal, 17 (1979), pp. 1030-1037.

[12] E. F. Toro, Riemann Solvers and Numerical Methods for Fluid Dynamics, a Practical Introduction, Springer, Berlin, 1997.

[13] R. M. Visbal And D. V. Gaitonde, On the use of higher-order finite-difference schemes on curvilinear and deforming meshes, Journal of Computational Physics, 181 (2002), pp. 155185.

[14] H. Zhang, M. Reggio, T. Y. Trépanier, and R. Camarero, Discrete form of the GCL for moving meshes and its implementation in CFD schemes, Computers and Fluids, 22 (1993), pp. $9-23$. 


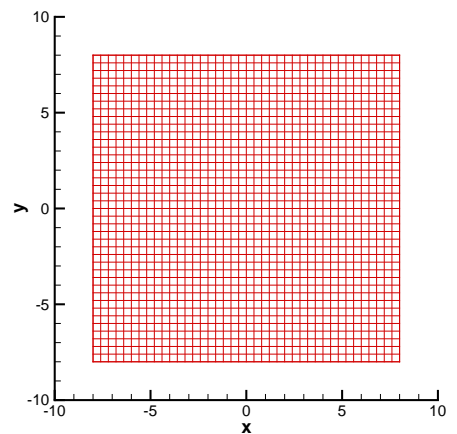

(a) grid

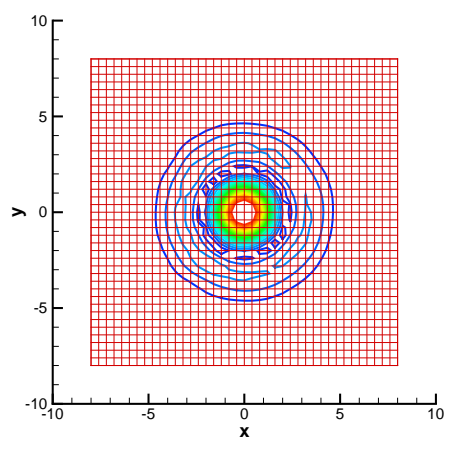

(c) S-LF

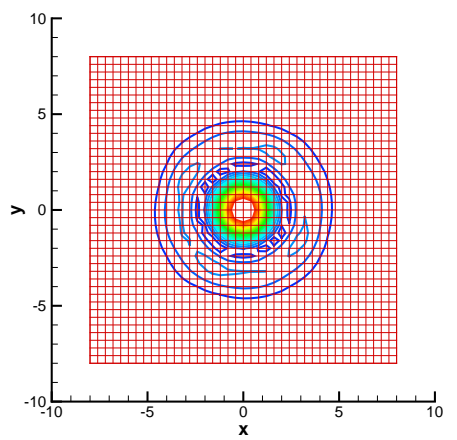

(e) A-LF

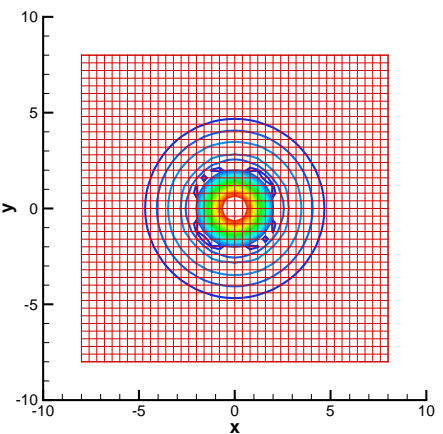

(b) initial condition

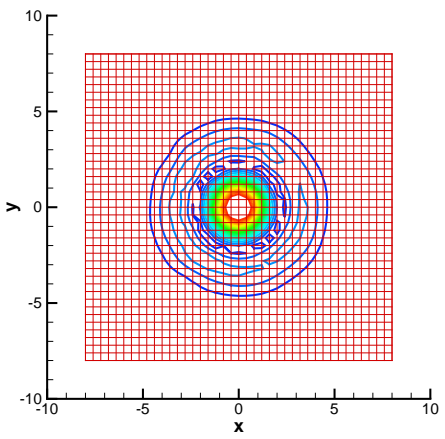

(d) S-R

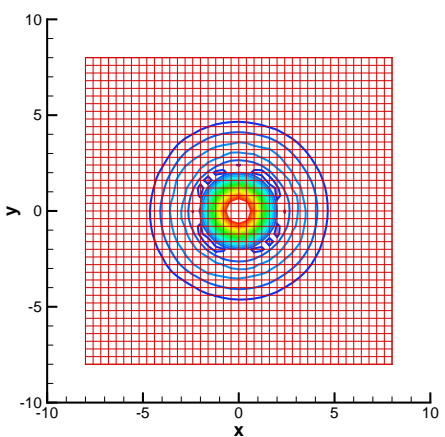

(f) A-HLLC

FIG. 5. Vorticity magnitude distribution of the results of two-dimensional moving vortex problem on the coarsest uniform grid. Here, 21 contours from 0.0 to 6.0 are shown. 


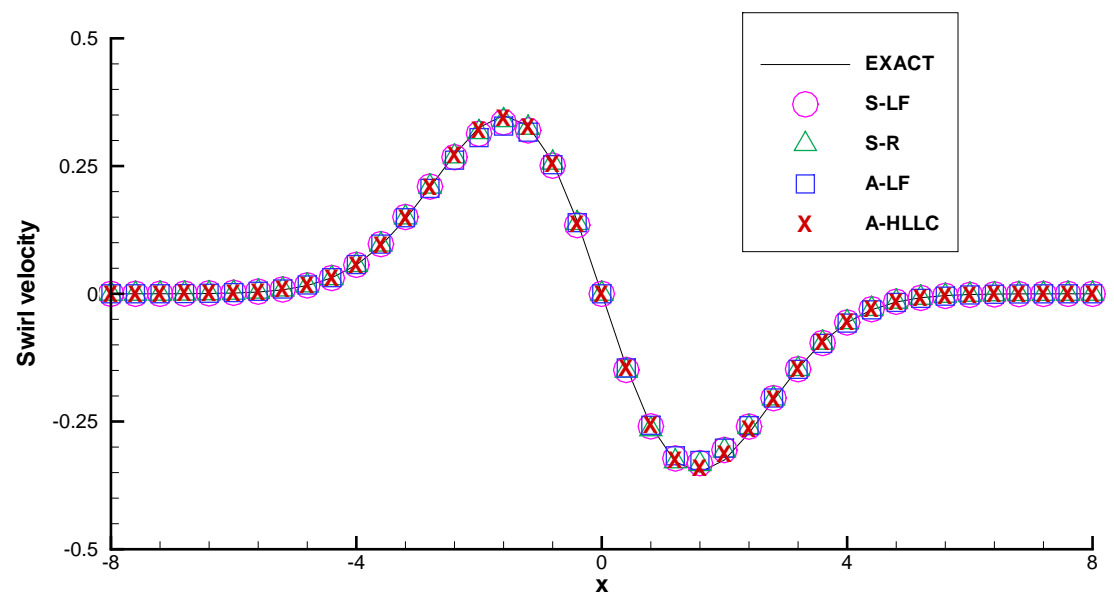

FIG. 6. Swirl velocity distributions on the line approximately passing through the vortex center of the two-dimensional moving vortex problem on the coarsest uniform grid. 


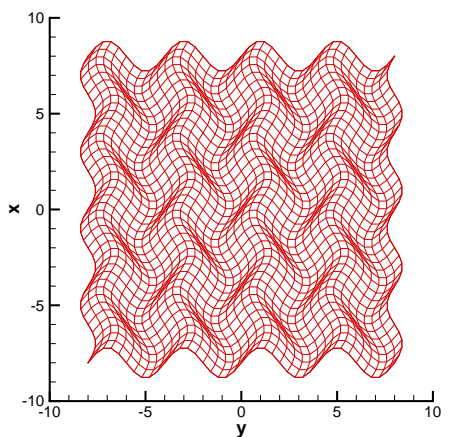

(a) grid

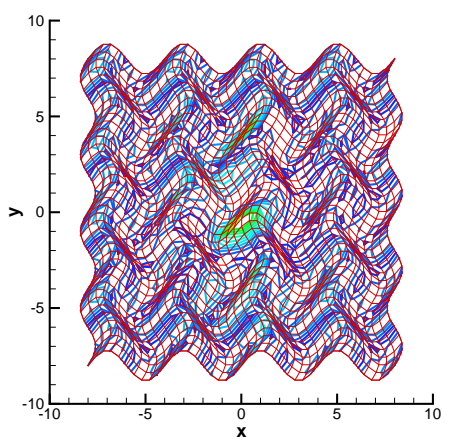

(c) S-LF

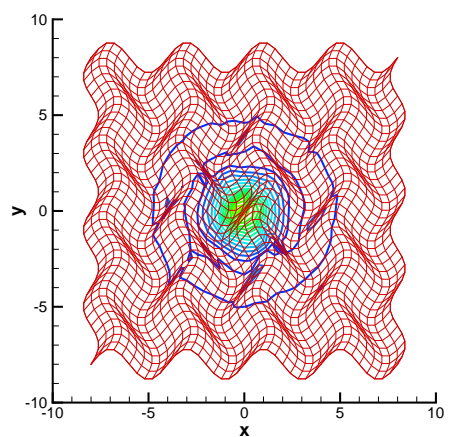

(e) A-LF

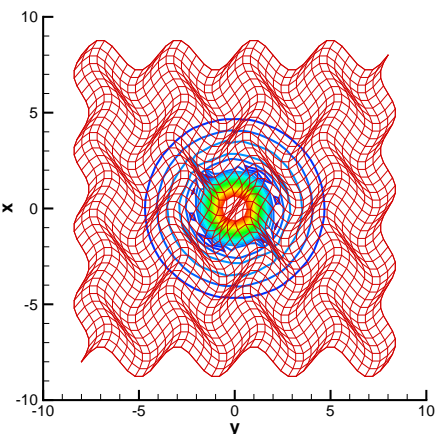

(b) initial condition

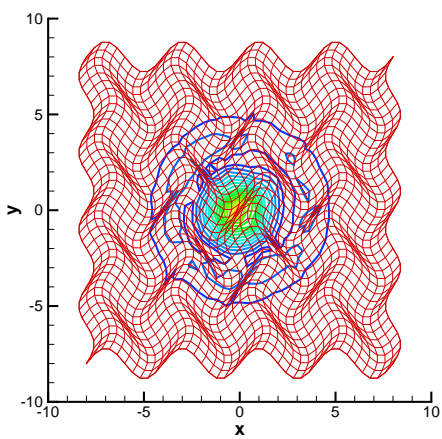

(d) S-R

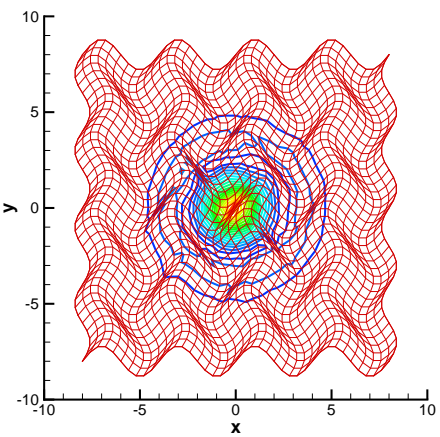

(f) A-HLLC

FIG. 7. Vorticity magnitude distribution of the results of two-dimensional moving vortex problem on the coarsest wavy grid. Here, 21 contours from 0.0 to 6.0 are shown. 


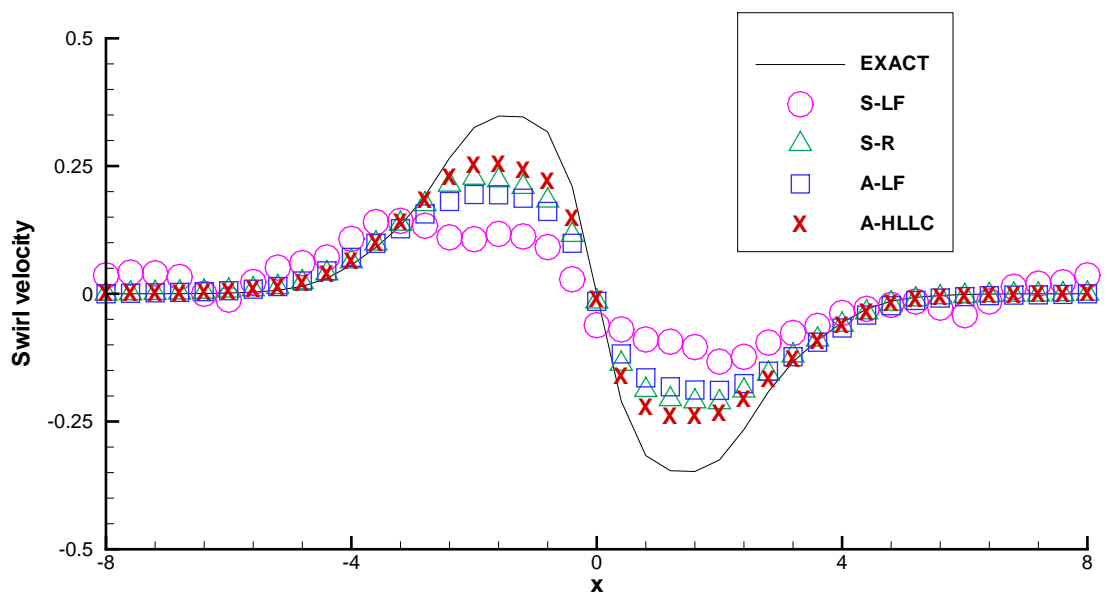

FIG. 8. Swirl velocity distributions on the line approximately passing through the vortex center of the two-dimensional moving vortex problem on the coarsest wavy grid. 


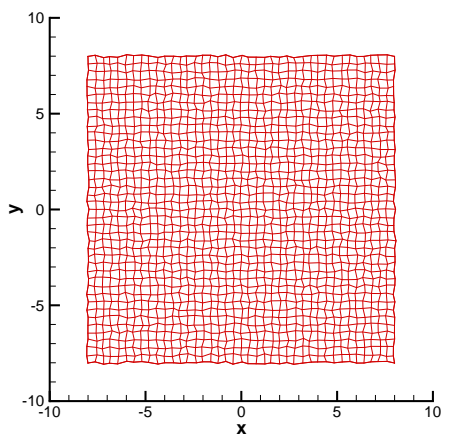

(a) grid

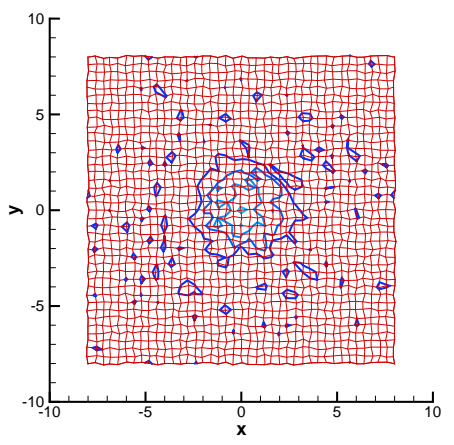

(c) S-LF

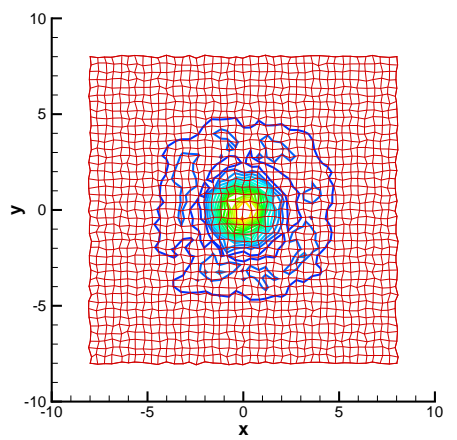

(e) A-LF

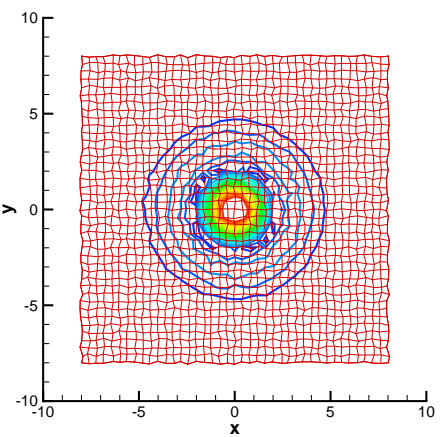

(b) initial condition

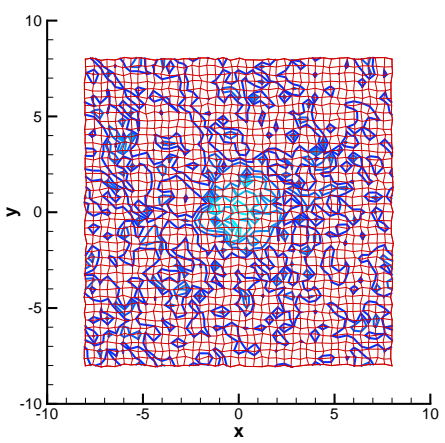

(d) S-R

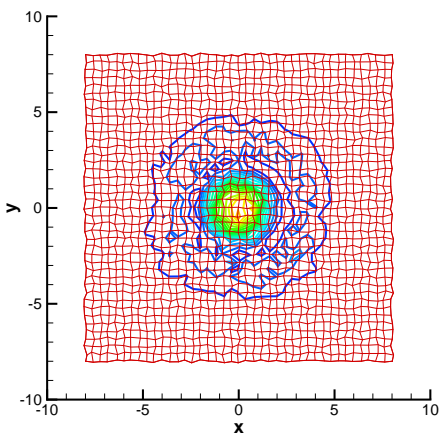

(f) A-HLLC

FIG. 9. Vorticity magnitude distribution of the results of two-dimensional moving vortex problem on the random grid. Here, 21 contours from 0.0 to 6.0 are shown. 


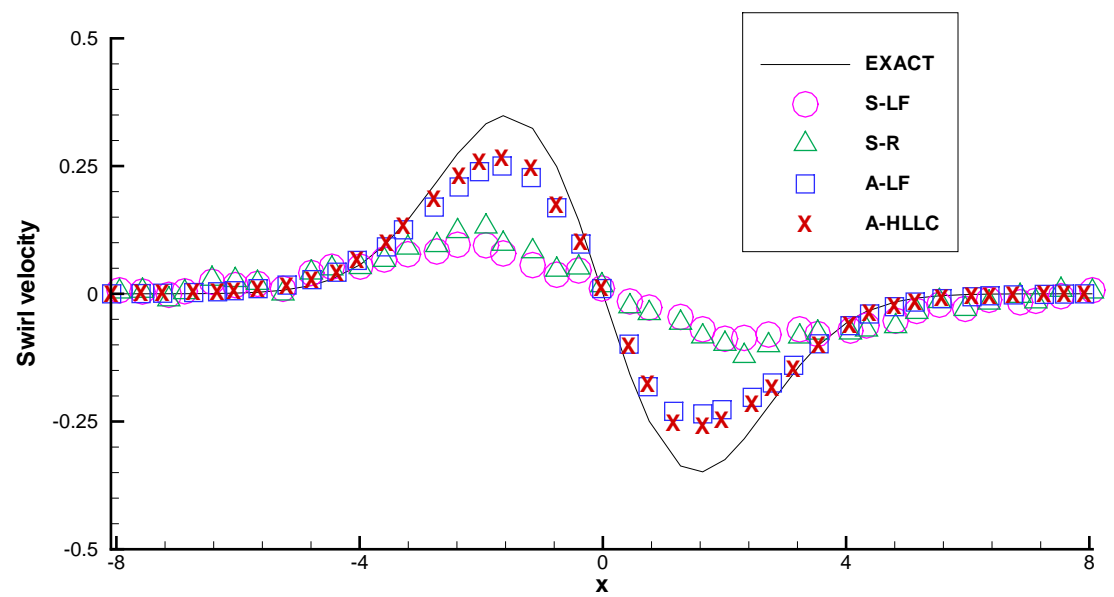

FIG. 10. Swirl velocity distributions on the line approximately passing through the vortex center of the two-dimensional moving vortex problem on the random grid. 


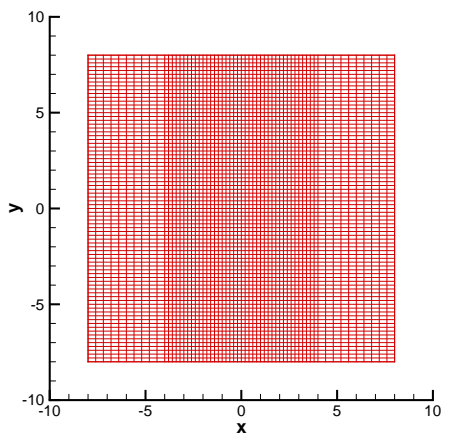

(a) grid

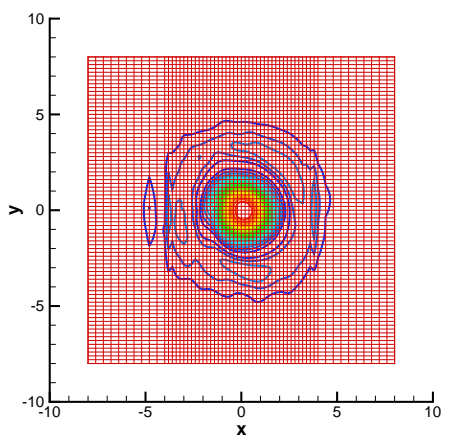

(c) S-LF

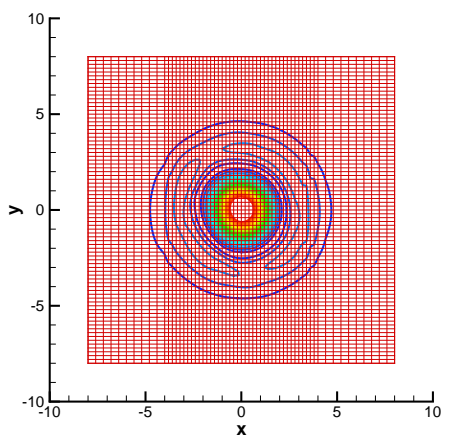

(e) A-LF

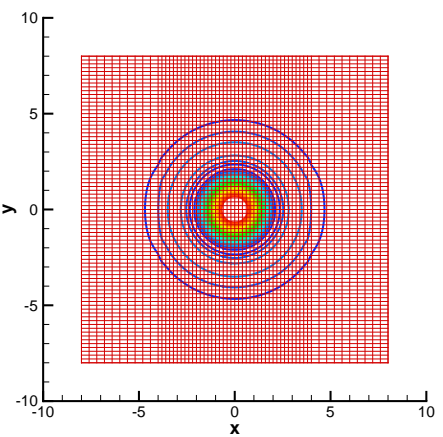

(b) initial condition

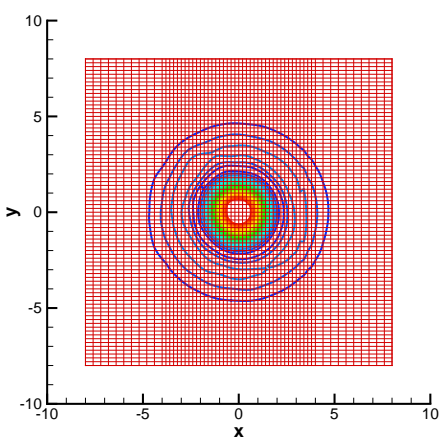

(d) S-R

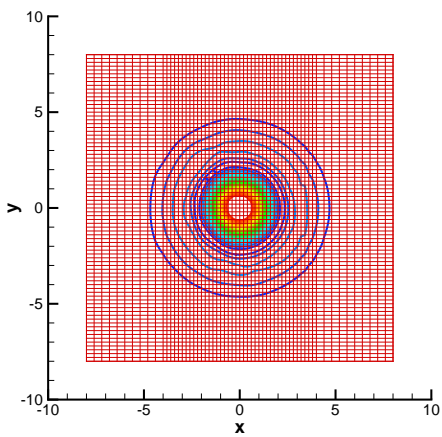

(f) A-HLLC

FIG. 11. Vorticity magnitude distribution of the results of two-dimensional moving vortex problem on a mesh with sudden jumps in grid spacing. Here, 21 contours from 0.0 to 6.0 are shown. 


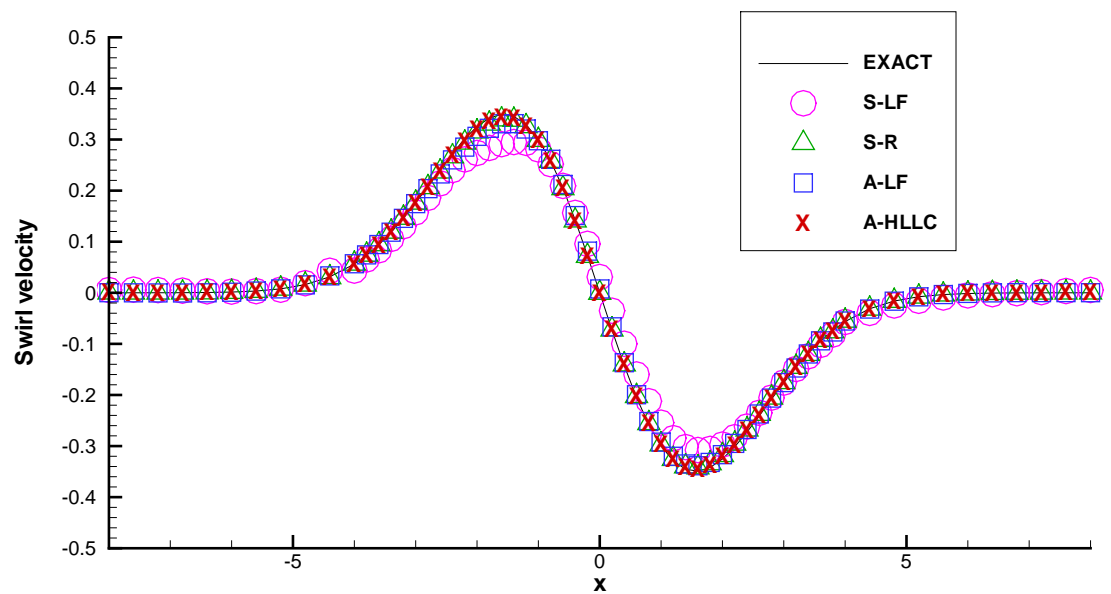

FIG. 12. Swirl velocity distributions on the line approximately passing through the vortex center of the two-dimensional moving vortex problem on a mesh with sudden jumps in grid spacing. 


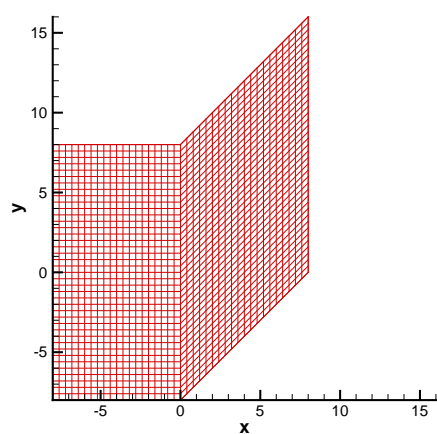

(a) grid

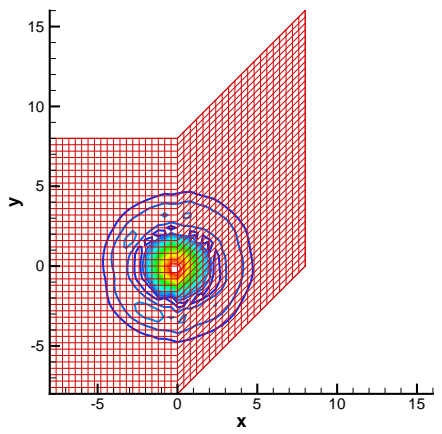

(c) S-LF

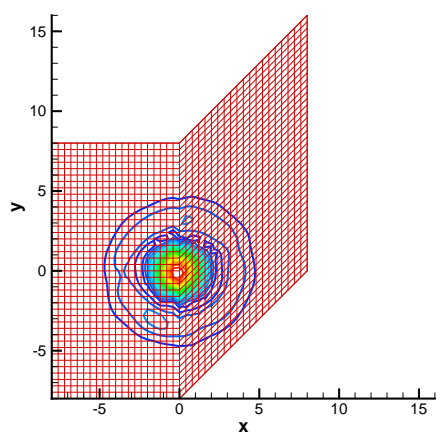

(e) A-LF

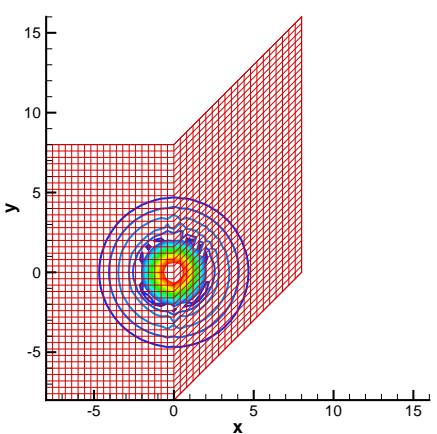

(b) initial condition

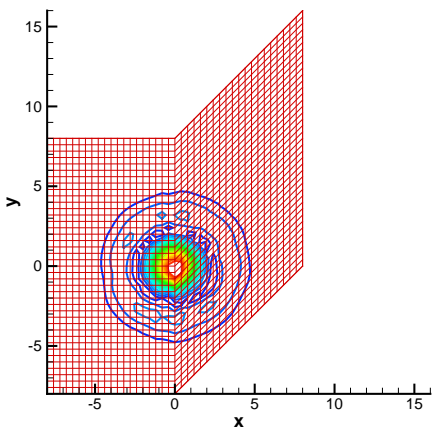

(d) S-R

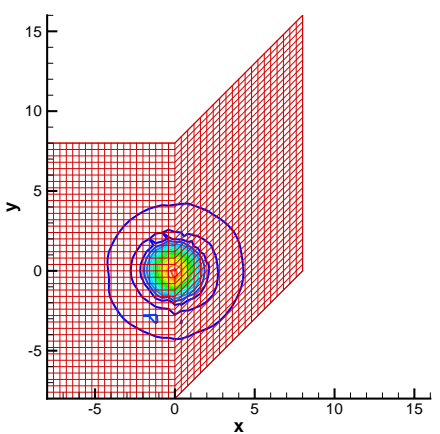

(f) A-HLLC

FIG. 13. Vorticity magnitude distribution of the results of two-dimensional moving vortex problem on a mesh with localized abrupt change. Here, 21 contours from 0.0 to 6.0 are shown. 


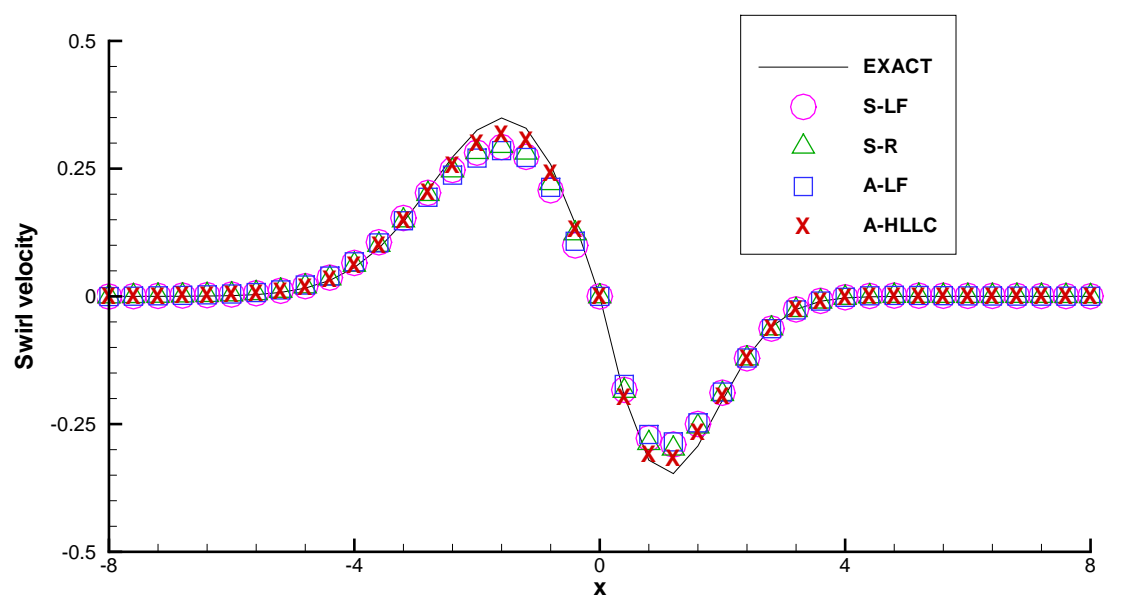

FIG. 14. Swirl velocity distributions on the line approximately passing through the vortex center of the two-dimensional moving vortex problem on a mesh with localized abrupt change.

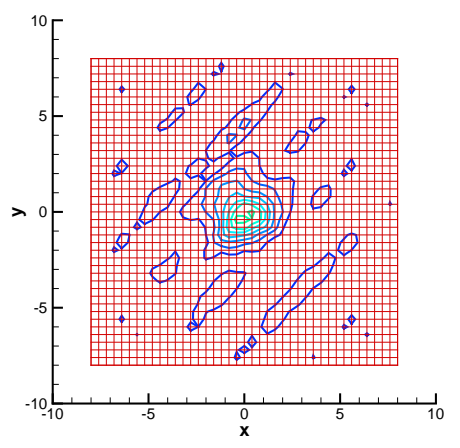

(a) S-LF

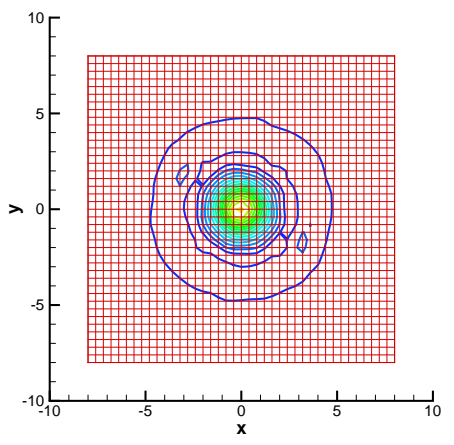

(c) A-LF

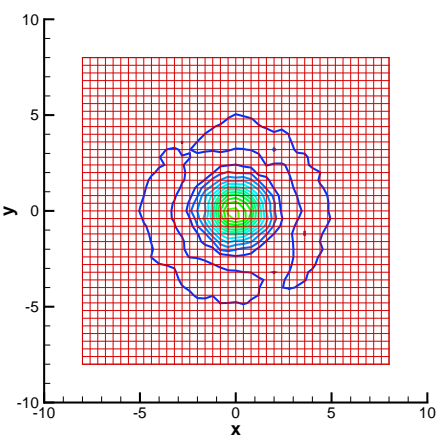

(b) S-R

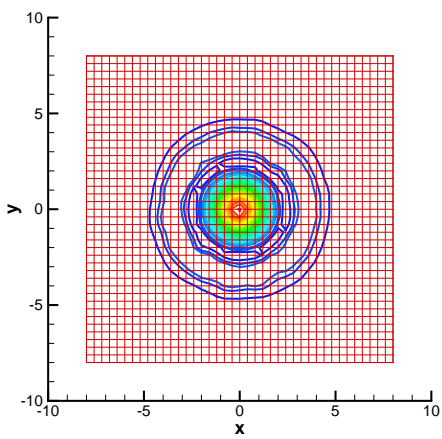

(d) A-HLLC

FIG. 15. Vorticity magnitude distribution of the results of the two-dimensional moving vortex problem on the coarsest dynamically changing grid. Here, 21 contours from 0.0 to 6.0 are shown. 


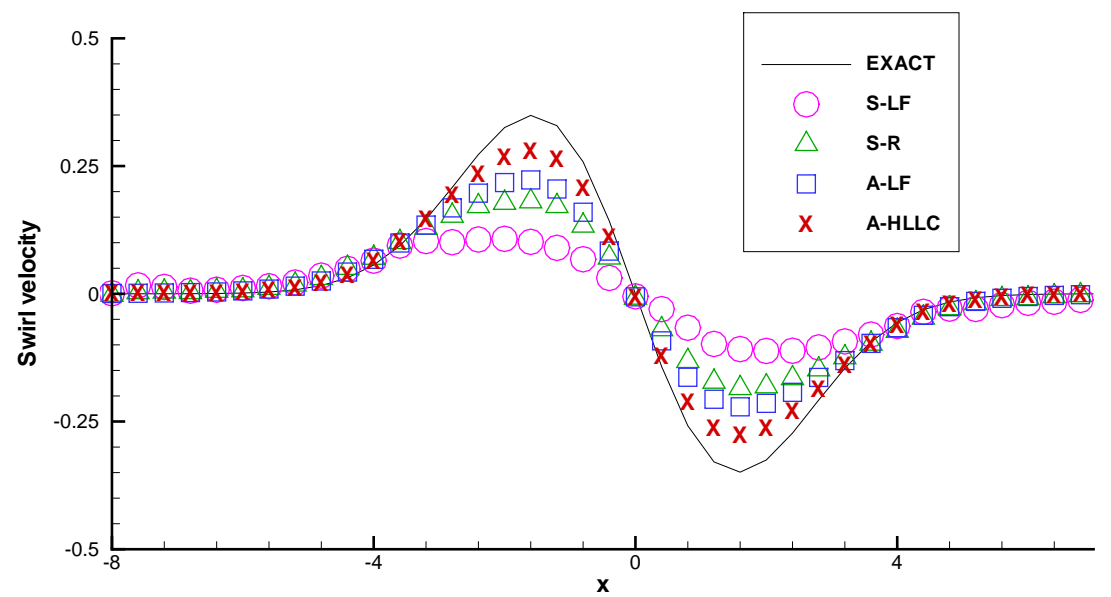

FIG. 16. Swirl velocity distributions on the line approximately passing through the vortex center of the two-dimensional moving vortex problem on the coarsest dynamically changing grid. 


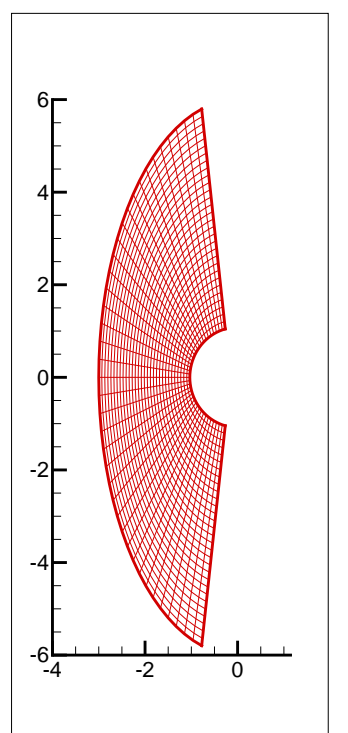

(a) physical grid $30 \times 40$

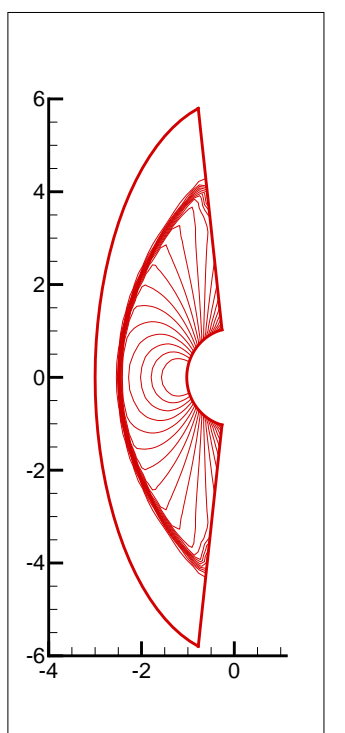

(b) S-LF

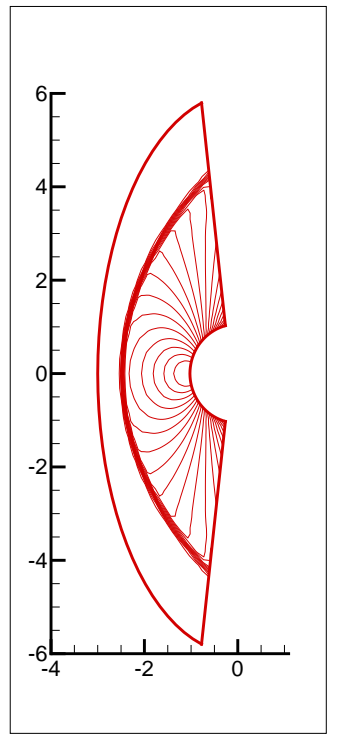

(c) S-R

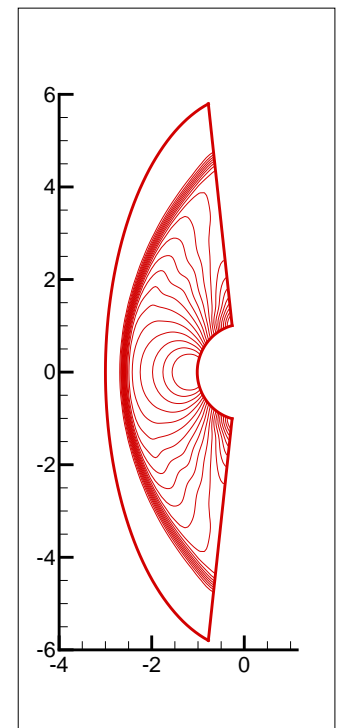

(d) A-LF

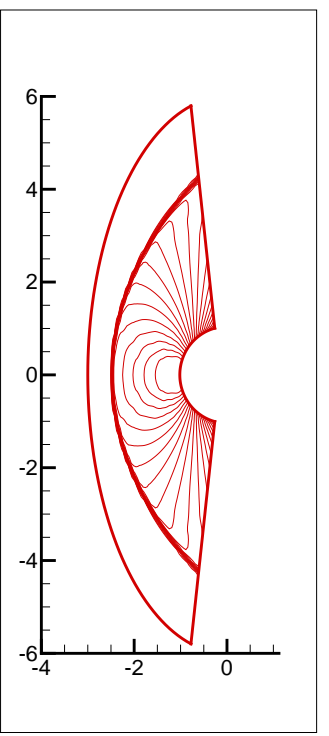

(e) A-HLLC

FIG. 17. Flow past a cylinder. (a) an illustration of the physical grid (drawing every other grid line); (b)-(e) pressure on the mesh $60 \times 80$. 